Supporting Information for

\title{
Capsanthin production in Escherichia coli by overexpression of capsanthin/capsorubin synthase from Capsicum annuum
}

\section{Authors}

Maiko Furubayashi ${ }^{*}$, Akiko Kubo ${ }^{2}$, Miho Takemura ${ }^{3}$, Yuko Otani $^{1}$, Takashi Maoka ${ }^{4}$, Yoshinobu Terada ${ }^{5}$, Katsuro Yaoi ${ }^{6}$, Kohji Ohdan², Norihiko Misawa ${ }^{3}$, Yasuo Mitani ${ }^{1}$

${ }^{1}$ Bioproduction Research Institute, National Institute of Advanced Industrial Science and Technology (AIST), Hokkaido, 062-8517, Japan

${ }^{2}$ Applied Research Laboratory, Ezaki Glico Co., Ltd., Osaka 555-8502, Japan

${ }^{3}$ Research Institute for Bioresources and Biotechnology, Ishikawa Prefectural University, Nonoichi, Ishikawa 921-8836, Japan

${ }^{4}$ Division of Food Function and Chemistry, Research Institute for Production Development, Kyoto, 606-0805, Japan

${ }^{5}$ Mechanism-based Research Laboratory, Ezaki Glico Co., Ltd., Osaka 555-8502, Japan

${ }^{6}$ Bioproduction Research Institute, National Institute of Advanced Industrial Science and Technology (AIST), Tsukuba, 305-8566, Japan

*Corresponding author. E-mail: maiko.furubayashi@aist.go.jp 

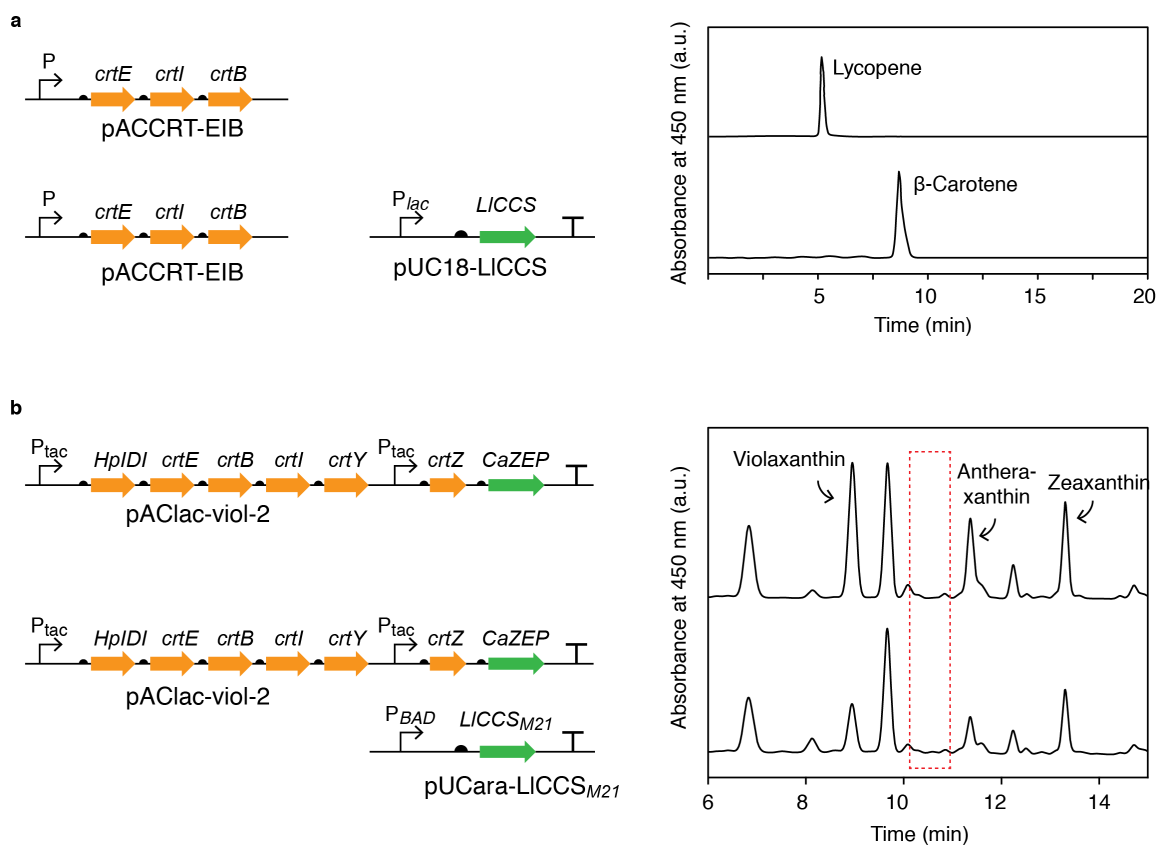

Figure S1. Carotenoid production using LICCS in E. coli. (a) LICCS expression in lycopene-producing $E$. coli. HPLC chromatogram of carotenoids extracted from $E$. coli harboring the indicated plasmids are shown. (b) LICCS expression in violaxanthin-producing $E$. coli. The violaxanthin operon construct is identical to that in Figure 3a. HPLC chromatogram of carotenoids extracted from E. coli harboring indicated plasmids are shown. The dotted red box indicates where the capsanthin peak could be expected. All assigned carotenoids were identified by retention time, absorbance spectrum and comparision with the known carotenoid standard.

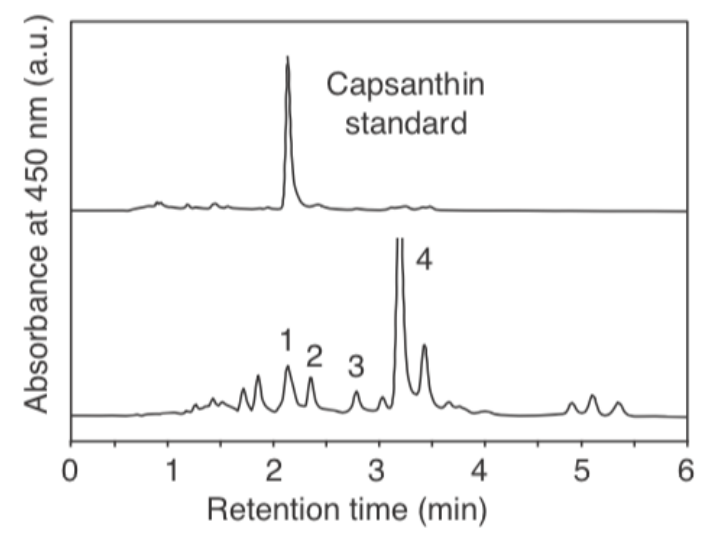

Figure S2. HPLC analysis of carotenoid extract from E. coli harboring pACHP-Zea and pUCara$\mathrm{CaCCS}_{\mathrm{M} 40}$-CaZEP (bottom) and capsanthin standard (top). 


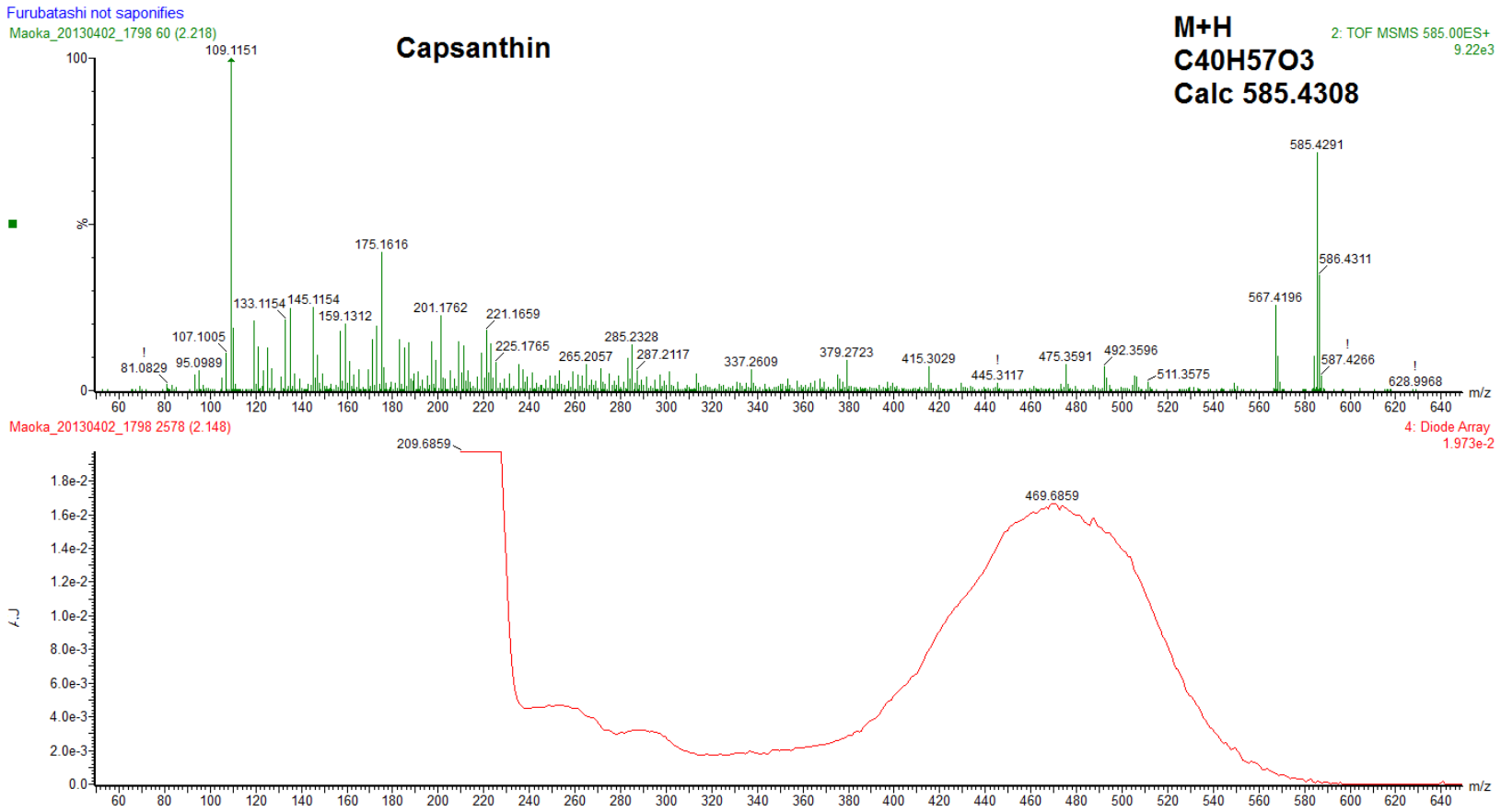

Figure S3. TOF-MS/MS and absorbance spectrum of peak 1 in Figure S2; capsanthin.

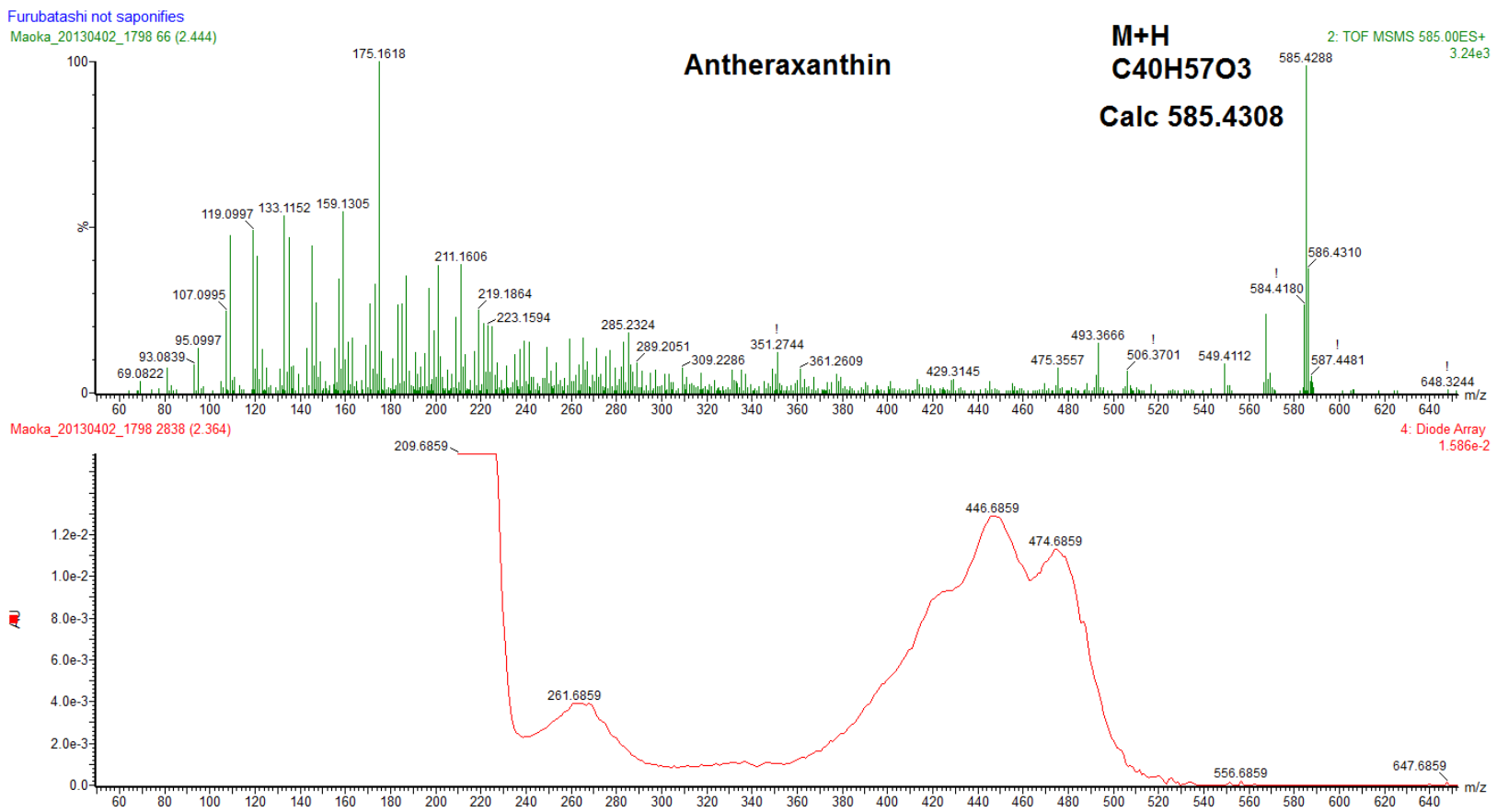

Figure S4. TOF-MS/MS and absorbance spectrum of peak 2 in Figure S2; antheraxanthin. 


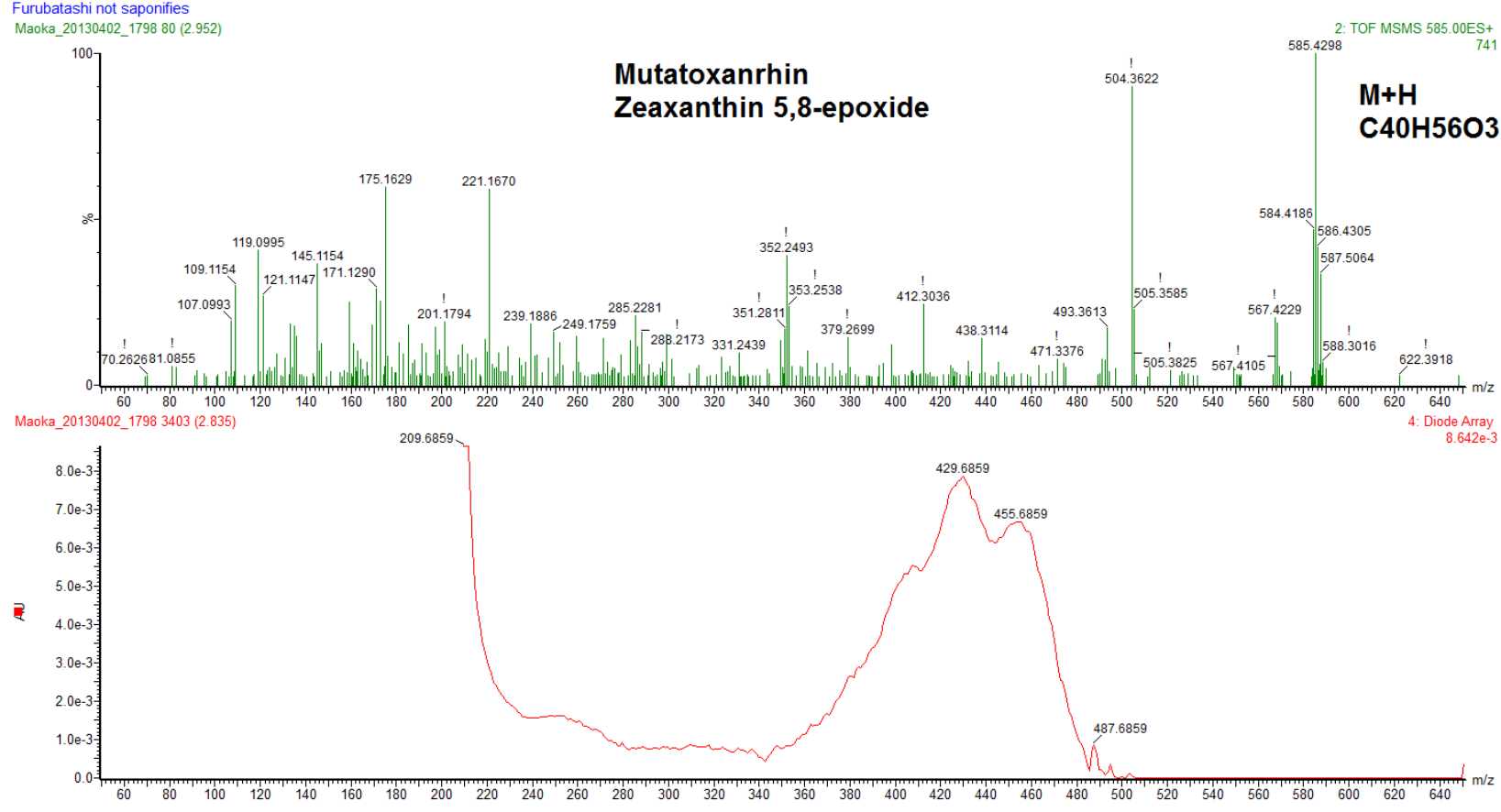

Figure S5. TOF-MS/MS and absorbance spectrum of peak 3 in Figure S2; mutatoxanthin.

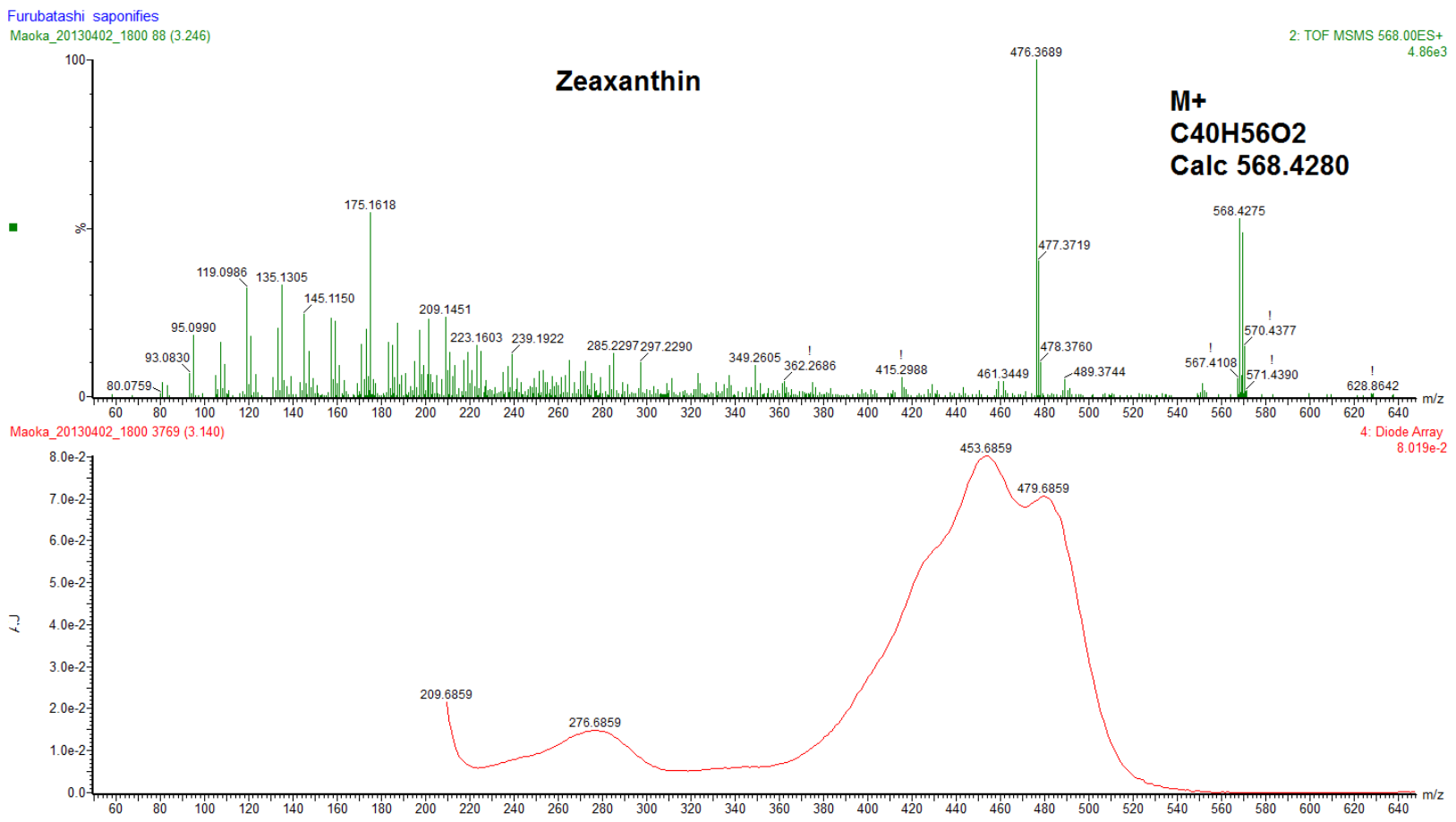

Figure S6. TOF-MS/MS and absorbance spectrum of peak 4 in Figure S2; zeaxanthin. 


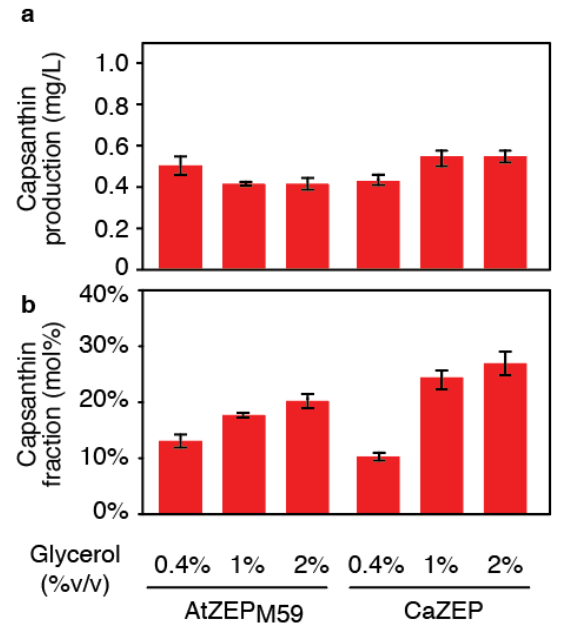

Figure S7. Optimizing of glycerol concentration for capsanthin production

The production and mol fractions among total carotenoids (panel $\mathbf{a}$ and $\mathbf{b}$, respectively) of capsanthin from $E$. coli strains cultured in different glycerol concentration \% (v/v). The label "AtZEP ${ }_{M 59}$ " indicates the $E$. coli strain harboring pACHP-Zea and pUClac-CaCCS ${ }_{M 40}-A_{\text {AtZEP }}{ }_{\text {M59, }}$ while "CaZEP" indicates the strain harboring pACHP-Zea and pUClac-CaCCS ${ }_{\mathrm{M} 40}-\mathrm{CaZEP}$.
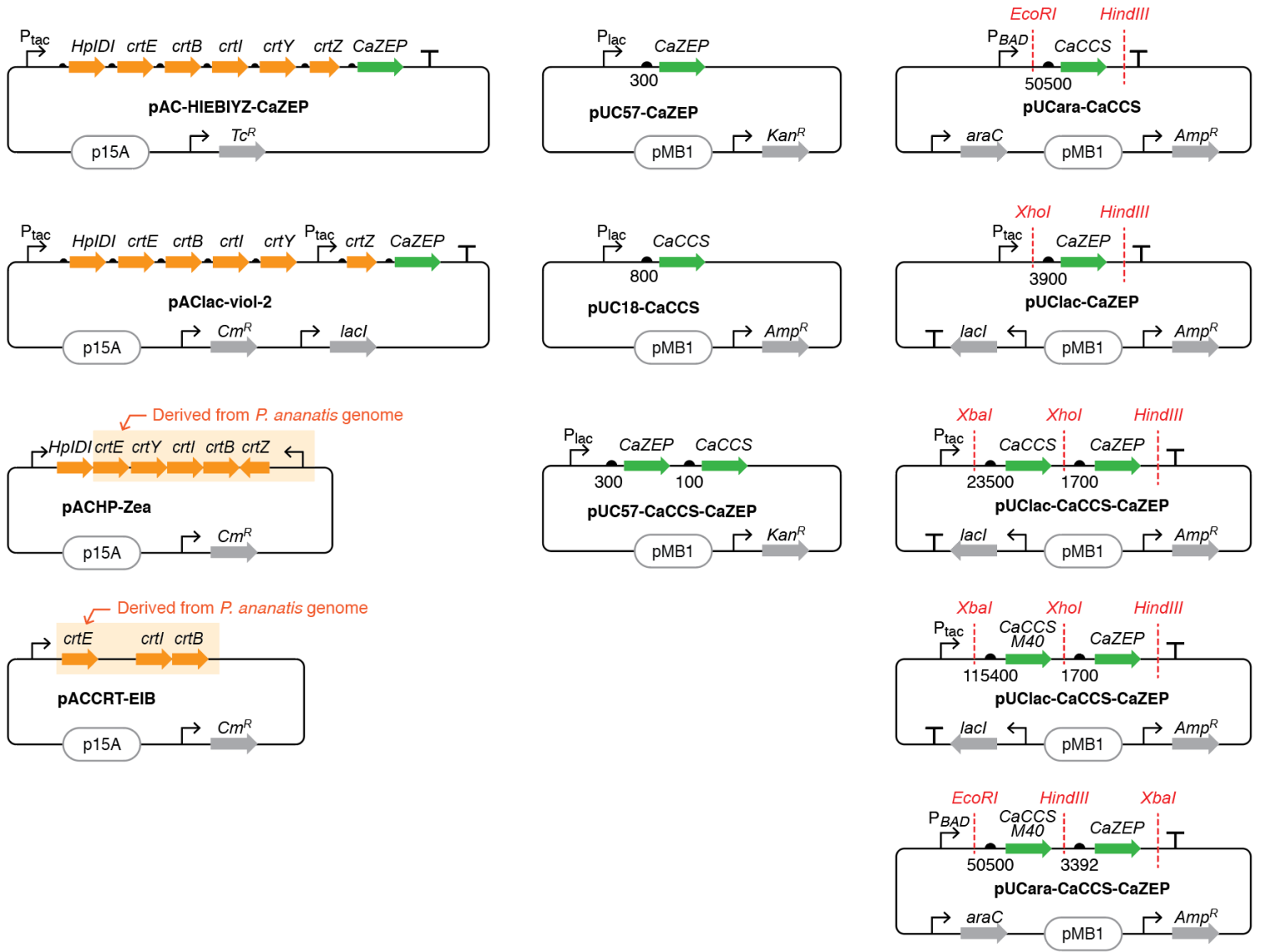

Figure S8. Maps of plasmids used in this study. The number below the RBS (black half-circle) on pUC plasmids indicates the TIR score calculated by RBS calculator (see main text). 
Table S1. Plasmids used in this study

\begin{tabular}{|c|c|c|}
\hline Plasmid name & $\begin{array}{l}\text { Vector backbone, ori, } \\
\text { marker }\end{array}$ & Source \\
\hline pUClac & $\mathrm{pMB1}, \mathrm{Amp}^{\mathrm{R}}$ & This study \\
\hline pACHP-Zea & pACYC184, p15A, Tc ${ }^{R}$ & Takemura et al. ${ }^{1}$ \\
\hline pACCRT-EBI & pACYC184, p15A, Cm ${ }^{R}$ & Misawa et al. ${ }^{2}$ \\
\hline PAC-HIEBIYZ-CaZEP & pACYC184, p15A, Tc ${ }^{R}$ & This study \\
\hline pAClac-viol-2 & pACYC184, p15A, Cm ${ }^{R}$ & This study \\
\hline pUCara-CaCCS & pUCara $^{3}, \mathrm{pMB} 1, \mathrm{Amp}^{\mathrm{R}}$ & This study \\
\hline pUCara-LICCS & pUCara $^{3}, \mathrm{pMB}, \mathrm{Amp}^{\mathrm{R}}$ & This study \\
\hline pUC57-CaCCS & pUC57-Kan, pMB1, Kan & This study \\
\hline pUC18-CaZEP & pUC18, pMB1, Amp ${ }^{R}$ & This study \\
\hline pUC18-CaZEP-CaCCS & pUC18, pMB1, Amp ${ }^{R}$ & This study \\
\hline pUClac-[ZEP] ${ }^{a}$ & pUClac, pMB1, Amp ${ }^{R}$ & This study \\
\hline pUClac- $[\mathrm{CaCCS}]^{\mathrm{b}}-[\mathrm{ZEP}]^{\mathrm{a}}$ & pUClac, pMB1, Amp ${ }^{R}$ & This study \\
\hline pUCara- $[\mathrm{CaCCS}]^{\mathrm{b}}-[\mathrm{ZEP}]^{\mathrm{a}}$ & pUCara, pMB1, Amp ${ }^{R}$ & This study \\
\hline
\end{tabular}

a) [ZEP] indicates either CaZEP, CaZEP ${ }_{M 56}$, AtZEP, AtZEP ${ }_{M 59}$

b) $[\mathrm{CaCCS}]$ indicates either $\mathrm{CaCCS}, \mathrm{CaCCS}_{\mathrm{M} 40}$

Table S2. Combination of plasmids used in each figure.

\begin{tabular}{|c|c|c|c|}
\hline Figure & Plasmid 1 & Plasmid 2 & Plasmid 3 \\
\hline \multirow[t]{2}{*}{ Figure $2 a$} & pACCRT-EIB & & \\
\hline & pACCRT-EIB & pUC18-CaCCS & \\
\hline \multirow[t]{3}{*}{ Figure 2c } & pACHP-Zea & pUC57-CaZEP & \\
\hline & pACHP-Zea & pUC57-CaZEP & pUC18-CaCCS \\
\hline & pACHP-Zea & pUC57-CaZEP-CaCCS & \\
\hline \multirow[t]{3}{*}{ Figure 3a } & pAClac-viol-2 & & \\
\hline & pAClac-viol-2 & pUCara-CaCCS ${ }_{M 40}$ & \\
\hline & pAClac-viol-2 & pUCara-CaCCS ${ }_{M 40}-\mathrm{CaZEP}$ & \\
\hline \multirow[t]{4}{*}{ Figure $3 b$} & pACHP-Zea & pUClac-CaZEP & \\
\hline & pACHP-Zea & pUClac-CaCCS-CaZEP & \\
\hline & pACHP-Zea & pUClac-CaCCS ${ }_{\mathrm{M} 40}$-CaZEP & \\
\hline & pACHP-Zea & pUCara-CaCCS ${ }_{M 40}$-CaZEP & \\
\hline Figure $3 c$ & pACHP-Zea & pUClac- $\mathrm{P}_{\mathrm{T} 7}-\mathrm{CaCCS}_{\mathrm{M} 40}-\mathrm{CaZEP}$ & \\
\hline \multirow[t]{2}{*}{ Figure 4} & pACHP-Zea & pUClac-[ZEP] ${ }^{a}$ & \\
\hline & pACHP-Zea & pUClac-CaCCS ${\mathrm{M} 40^{-}}^{-}[\mathrm{ZEP}]^{\mathrm{a}}$ & \\
\hline \multirow[t]{4}{*}{ Figure S1 } & pACCRT-EIB & & \\
\hline & pACCRT-EIB & pUC18-LICCS & \\
\hline & pAClac-viol-2 & & \\
\hline & pAClac-viol-2 & pUCara-LICCS ${ }_{\mathrm{M} 21}$ & \\
\hline Figure S2 & pACHP-Zea & pUCara-CaCCS ${ }_{M 40}$-CaZEP & \\
\hline \multirow[t]{2}{*}{ Figure S7 } & pACHP-Zea & pUClac-CaCCS ${ }_{M 40}-$ AtZEP $_{M 59}$ & \\
\hline & pACHP-Zea & pUClac-CaCCS ${ }_{M 40}-\mathrm{CaZEP}$ & \\
\hline
\end{tabular}

a) [ZEP] indicates either CaZEP, CaZEP ${ }_{M 56}$, AtZEP $_{\text {, AtZEP }}$ M59 
Table S3. DNA sequences of violaxanthin operon plasmids.

\begin{tabular}{l}
\hline Name \\
\hline pAC-HIEBIYZ- \\
CaZEP
\end{tabular}

\section{Sequence}

AGCTGTTGACAATTAATCATCGGCTCGTATAATGTGTGGAATTGTGAGCGGATAACAATTTCACACAGGAAACACTCGAGGAGGCAGCTATGCTTCGTTCGTTGCT CAGAGGCCTCACGCATTTCCCCCGCGTGAACTCCGCGCAGCAGCCCAGCTGTGCACACGCGCGACTCCAGTTTAGGCCCAGAAGCATGCAGCTGCTTGCCGAGGA CCGCACAGACCATATGAGGGGTGCAAGTACCTGGGCAGGCGGGCAGTCGCAGGATGAGCTGATGCTGAAGGACGAGTGCATCTTGGTGGATGCTGACGACAAC ATTACAGGCCATGTCAGCAAGCTGGAGTGCCACAAGTTCCTACCACATCAGCCTGCAGGCCTGCTGCACCGGGCCTTCTCTGTATTCCTGTTTGACGACCAGGGGC GACTGCTGCTGCAACAGCGTGCACGATCAAAAATCACATTCCCCAGTGTGTGGACCAACACCTGCTGCAGCCACCCTCTACATGGGCAGACCCCAGATGAGGTGG ACCAACTAAGCCAGGTGGCCGACGGCACAGTACCTGGCGCAAAGGCTGCTGCCATCCGCAAGTTGGAGCACGAGCTGGGGATACCAGCGCACCAGCTGCCGGCC AGCGCGTTTCGCTTCCTCACGCGTTTGCACTACTGCGCCGCGGACGTGCAGCCGGCTGCGACACAATCAGCACTCTGGGGCGAGCACGAAATGGACTACATCTTAT TCATCCGGGCCAACGTCACCCTTGCGCCCAACCCTGACGAGGTGGACGAAGTCAGGTACGTGACGCAGGAGGAGCTGCGGCAGATGATGCAGCCGGACAATGGG TTGCAATGGTCGCCGTGGTTTCGCATCATCGCCGCGCGCTTCCTTGAGCGCTGGTGGGCTGACCTAGACGCGGCCCTGAACACTGACAAACACGAGGATTGGGGA ACGGTGCATCACATCAACGAAGCGTGAGATCCAGGAGGCAGCTATGACGGTCTGCGCAAAAAAACACGTTCATCTCACTCGCGATGCTGCGGAGCAGTTACTGGC TGATATTGATCGACGCCTTGATCAGTTATTGCCCGTGGAGGGAGAACGGGATGTTGTGGGTGCCGCGATGCGTGAAGGTGCGCTGGCACCGGGAAAACGTATTC GCCCCATGTTGCTGTTGCTGACCGCCCGCGATCTGGGTTGCGCTGTCAGCCATGACGGATTACTGGATTTGGCCTGTGCGGTGGAAATGGTCCACGCGGCTTCGCT GATCCTTGACGATATGCCCTGCATGGACGATGCGAAGCTGCGGCGCGGACGCCCTACCATTCATTCTCATTACGGAGAGCATGTGGCAATACTGGCGGCGGTTGC CTTGCTGAGTAAAGCCTTTGGCGTAATTGCCGATGCAGATGGCCTCACGCCGCTGGCAAAAAATCGGGCGGTTTCTGAACTGTCAAACGCCATCGGCATGCAAGG ATTGGTTCAGGGTCAGTTCAAGGATCTGTCTGAAGGGGATAAGCCGCGCAGCGCTGAAGCTATTTTGATGACGAATCACTTTAAAACCAGCACGCTGTTTTGTGCC TCCATGCAGATGGCCTCGATTGTTGCGAATGCCTCCAGCGAAGCGCGTGATTGCCTGCATCGTTTTTCACTTGATCTTGGTCAGGCATTTCAACTGCTGGACGATTT GACCGATGGCATGACCGACACCGGTAAGGATAGCAATCAGGACGCCGGTAAATCGACGCTGGTCAATCTGTTAGGCCCGAGGGCGGTTGAAGAACGTCTGAGAC AACATCTTCAGCTTGCCAGTGAGCATCTCTCTGCGGCCTGCCAACACGGGCACGCCACTCAACATTTTATTCAGGCCTGGTTTGACAAAAAACTCGCTGCCGTCAGT TAAGATCCAGGAGGCAGCTATGAATAATCCGTCGTTACTCAATCATGCGGTCGAAACGATGGCAGTTGGCTCGAAAAGTTTTGCGACAGCCTCAAAGTTATTTGAT GCAAAAACCCGGCGCAGCGTACTGATGCTCTACGCCTGGTGCCGCCATTGTGACGATGTTATTGACGATCAGACGCTGGGCTTTCAGGCCCGGCAGCCTGCCTTAC AAACGCCCGAACAACGTCTGATGCAACTTGAGATGAAAACGCGCCAGGCCTATGCAGGATCGCAGATGCACGAACCGGCGTTTGCGGCTTTTCAGGAAGTGGCTA TGGCTCATGATATCGCCCCGGCTTACGCGTTTGATCATCTGGAAGGCTTCGCCATGGATGTACGCGAAGCGCAATACAGCCAACTGGATGATACGCTGCGCTATTG CTATCACGTTGCAGGCGTTGTCGGCTTGATGATGGCGCAAATCATGGGCGTGCGGGATAACGCCACGCTGGACCGCGCCTGTGACCTTGGGCTGGCATTTCAGTT GACCAATATTGCTCGCGATATTGTGGACGATGCGCATGCGGGCCGCTGTTATCTGCCGGCAAGCTGGCTGGAGCATGAAGGTCTGAACAAAGAGAATTATGCGGC ACCTGAAAACCGTCAGGCGCTGAGCCGTATCGCCCGTCGTTTGGTGCAGGAAGCAGAACCTTACTATTTGTCTGCCACAGCCGGCCTGGCAGGGTTGCCCCTGCGT TCCGCCTGGGCAATCGCTACGGCGAAGCAGGTTTACCGGAAAATAGGTGTCAAAGTTGAACAGGCCGGTCAGCAAGCCTGGGATCAGCGGCAGTCAACGACCAC GCCCGAAAAATTAACGCTGCTGCTGGCCGCCTCTGGTCAGGCCCTTACTTCCCGGATGCGGGCTCATCCTCCCCGCCCTGCGCATCTCTGGCAGCGCCCGCTCTAGC AATTCAGGAGGCAGCTATGAAACCAACTACGGTAATTGGTGCAGGCTTCGGTGGCCTGGCACTGGCAATTCGTCTACAAGCTGCGGGGATCCCCGTCTTACTGCTT GAACAACGTGATAAACCCGGCGGTCGGGCTTATGTCTACGAGGATCAGGGGTTTACCTTTGATGCAGGCCCGACGGTTATCACCGATCCCAGTGCCATTGAAGAA CTGTTTGCACTGGCAGGAAAACAGTTAAAAGAGTATGTCGAACTGCTGCCGGTTACGCCGTTTTACCGCCTGTGTTGGGAGTCAGGGAAGGTCTTTAATTACGATA ACGATCAAACCCGGCTCGAAGCGCAGATTCAGCAGTTTAATCCCCGCGATGTCGAAGGTTATCGTCAGTTTCTGGACTATTCACGCGCGGTGTTTAAAGAAGGCTA TCTAAAGCTCGGTACTGTCCCTTTTTTATCGTTCAGAGACATGCTTCGCGCCGCACCTCAACTGGCGAAACTGCAGGCATGGAGAAGCGTTTACAGTAAGGTTGCC AGTTACATCGAAGATGAACATCTGCGCCAGGCGTTTTCTTTCCACTCGCTGTTGGTGGGCGGCAATCCCTTCGCCACCTCATCCATTTATACGTTGATACACGCGCT GGAGCGTGAGTGGGGCGTCTGGTTTCCGCGTGGCGGCACCGGCGCATTAGTTCAGGGGATGATAAAGCTGTTTCAGGATCTGGGTGGCGAAGTCGTGTTAAACG CCAGAGTCAGCCATATGGAAACGACAGGAAACAAGATTGAAGCCGTGCATTTAGAGGACGGTCGCAGGTTCCTGACGCAAGCCGTCGCGTCAAATGCAGATGTG GTTCATACCTATCGCGACCTGTTAAGCCAGCACCCTGCCGCGGTTAAGCAGTCCAACAAACTGCAGACTAAGCGCATGAGTAACTCTCTGTTTGTGCTCTATTTTGG TTTGAATCACCATCATGATCAGCTCGCGCATCACACGGTTTGTTTCGGCCCGCGTTACCGCGAGCTGATTGACGAAATTTTTAATCATGATGGCCTCGCAGAGGACT TCTCACTTTATCTGCACGCGCCCTGTGTCACGGATTCGTCACTGGCGCCTGAAGGTTGCGGCAGTTACTATGTGTTGGCGCCGGTGCCGCATTTAGGCACCGCGAA CCTCGACTGGACGGTTGAGGGGCCAAAACTACGCGACCGTATTTTTGCGTACCTTGAGCAGCATTACATGCCTGGCTTACGGAGTCAGCTGGTCACGCACCGGAT GTTTACGCCGTTTGATTTTCGCGACCAGCTTAATGCCTATCATGGCTCAGCCTTTTCTGTGGAGCCCGTTCTTACCCAGAGCGCCTGGTTTCGGCCGCATAACCGCGA TAAAACCATTACTAATCTCTACCTGGTCGGCGCAGGCACGCATCCCGGCGCAGGCATTCCTGGCGTCATCGGCTCGGCAAAAGCGACAGCAGGTTTGATGCTGGA GGATCTGATATGAGATCCAGGAGGCAGCTATGCAACCGCATTATGATCTGATTCTCGTGGGGGCTGGACTCGCGAATGGCCTTATCGCCCTGCGTCTTCAGCAGCA GCAACCTGATATGCGTATTTTGCTTATCGACGCCGCACCCCAGGCGGGCGGGAATCATACGTGGTCATTTCACCACGATGATTTGACTGAGAGCCAACATCGTTGG ATAGCTCCGCTGGTGGTTCATCACTGGCCCGACTATCAGGTACGCTTTCCCACACGCCGTCGTAAGCTGAACAGCGGCTACTTTTGTATTACTTCTCAGCGTTTCGCT GAGGTTTTACAGCGACAGTTTGGCCCGCACTTGTGGATGGATACCGCGGTCGCAGAGGTTAATGCGGAATCTGTTCGGTTGAAAAAGGGTCAGGTTATCGGTGCC CGCGCGGTGATTGACGGGCGGGGTTATGCGGCAAATTCAGCACTGAGCGTGGGCTTCCAGGCGTTTATTGGCCAGGAATGGCGATTGAGCCACCCGCATGGTTT ATCGTCTCCCATTATCATGGATGCCACGGTCGATCAGCAAAATGGTTATCGCTTCGTGTACAGCCTGCCGCTCTCGCCGACCAGATTGTTAATTGAAGACACGCACT ATATTGATAATGCGACATTAGATCCTGAATGCGCGCGGCAAAATATTTGCGACTATGCCGCGCAACAGGGTTGGCAGCTTCAGACACTGCTGCGAGAAGAACAGG GCGCCTTACCCATTACTCTGTCGGGCAATGCCGACGCATTCTGGCAGCAGCGCCCCCTGGCCTGTAGTGGATTACGTGCCGGTCTGTTCCATCCTACCACCGGCTAT TCACTGCCGCTGGCGGTTGCCGTGGCCGACCGCCTGAGTGCACTTGATGTCTTTACGTCGGCCTCAATTCACCATGCCATTACGCATTTTGCCCGCGAGCGCTGGCA GCAGCAGGGCTTTTTCCGCATGCTGAATCGCATGCTGTTTTTAGCCGGACCCGCCGATTCACGCTGGCGGGTTATGCAGCGTTTTTATGGTTTACCTGAAGATTTAA TTGCCCGTTTTTATGCGGGAAAACTCACGCTGACCGATCGGCTACGTATTCTGAGCGGCAAGCCGCCTGTTCCGGTATTAGCAGCATTGCAAGCCATTATGACGAC TCATCGTTAAGATCCAGGAGGCAGCTATGTTGTGGATTTGGAATGCCCTGATCGTTTTCGTTACCGTGATTGGCATGGAAGTGATTGCTGCACTGGCACACAAATA CATCATGCACGGCTGGGGTTGGGGATGGCATCTTTCACATCATGAACCGCGTAAAGGTGCGTTTGAAGTTAACGATCTTTATGCCGTGGTTTTTGCTGCATTATCGA TCCTGCTGATTTATCTGGGCAGTACAGGAATGTGGCCGCTCCAGTGGATTGGCGCAGGTATGACGGCGTATGGATTACTCTATTTTATGGTGCACGACGGGCTGGT GCATCAACGTTGGCCATTCCGCTATATTCCACGCAAGGGCTACCTCAAACGGTTGTATATGGCGCACCGTATGCATCACGCCGTCAGGGGCAAAGAAGGTTGTGTT TCTTTTGGCTTCCTCTATGCGCCGCCCCTGTCAAAACTTCAGGCGACGCTCCGGGAAAGACATGGCGCTAGAGCGGGCGCTGCCAGAGATGCGCAGGGCGGGGA GGATGAGCCCGCATCCGGGAAGTAAGATCCAGGAGGCAGCTATGTATAGCACCGTGTTTTATACCAGCGTTCATCCGAGCACCAGCGTGTTTAGCCGCAAACAGC TGCCGCTGCTGATTAGCAAAGATTTTCCGGCCGAACTGTATCATAGCCTGCCGTGCAAAAGCCTGGAAAATGGTCATATTAAGAAAGTGAAAGGCGTTAAAGCCA CCCTGGCCGAAGCACCGGCCACCCCGACCGAAAAATCTAACAGCGAAGTGCCGCAGAAAAAACTGAAAGTGCTGGTTGCCGGCGGTGGCATTGGTGGCCTGGTG TTTGCGCTGGCGGCGAAAAGAAAGGCTTTGATGTGCTGGTTTTTGAACGTGATCTGAGCGCCATCCGCGGCGAAGGCCAGTATCGTGGCCCGATTCAGATCCAG AGCAATGCCCTGGCGGCCCTGGAAGCGATTGATATGGATGTGGCCGAAGAAATCATGAACGCGGGTTGCATTACCGGCCAGCGTATCAATGGTCTGGTTGATGGT ATTAGCGGCAACTGGTATTGTAAATTTGATACCTTTACCCCGGCCGTGGAACGCGGCCTGCCGGTGACCCGTGTTATTAGCCGCATGACCCTGCAGCAGATCCTGG CACGTGCCGTGGGTGAAGATGTTATTATGAACGAAAGCAATGTGGTTAACTTTGAAGATGATGGCGAAAAAGTGACCGTGGTTCTGGAAAATGGTCAGCGCTTTA CCGGCGATCTGCTGGTTGGTGCCGATGGCATCCGTAGCAAAGTGCGCACCAACCTGTTTGGTCATAGCGAAGCGACCTATAGCGGTTATACCTGTTATACCGGCAT TGCCGATTTTGTTCCGGCGGATATCGATACCGTGGGTTATCGTGTTTTTCTGGGCCATAAACAGTATTTTGTGAGCAGCGATGTTGGTGGCGGTAAAATGCAGTGG TATGCGTTTCATAATGAACCGGCCGGCGGTGTGGATGCCCCGAATGGCAAGAAAGAACGCCTGCTGAAAATTTTTGGCGGTTGGTGCGATAATGTGATCGATCTG CTGGTTGCCACCGATGAAGATGCGATTCTGCGTCGCGATATCTATGATCGTCCGCCGACCTTTAATTGGGGTCGTGGTCGTGTGACCCTGCTGGGTGATAGCGTTC ATGCCATGCAGCCGAACCTGGGTCAGGGCGGTTGTATGGCCATTGAAGATAGCTATCAGCTGGCGCTGGAACTGGAAAAAGCCTGGAGCCGCAGCGCCGAAAGC GGTAGCCCGATGGATGTTATCAGCAGCCTGCGTAGCTATGAAAGCGCCCGTAAACTGCGCGTGGGTGTTATTCATGGCCTGGCCCGTATGGCCGCAATCATGGCA AGCACCTATAAAGCGTATCTGGGCGTGGGTCTGGGTCCGCTGAGCTTTCTGACCAAATATCGTATTCCGCACCCGGGTCGTGTTGGCGGTCGTGTGTTTGTTGATC TGGGCATGCCGCTGATGCTGAGCTGGGTGCTGGGCGGTAATGGTGATAAACTGGAAGGCCGTATCCAGCATTGCCGCCTGAGCGAAAAAGCCAACGATCAGCTG CGTCGCTGGTTTGAAGATGATGATGCGCTGGAACGCGCCACCGATGCCGAATGGCTGCTGCTGCCGGCCGCAAATGGCAATAGCGCGCTGGAAACCATTGTGCTG AGCCGTGATGAAGATGTTCCGTGCACCATTGGTAGCGTGAGCCATACCAACATCCCGGGCAAAAGCGTGGTTCTGCCGCTGCCGCAGGTTAGCGAAATGCATGCC CAGATTAGCTGTAAAAACAATGCGTTTTTCGTGACCGATTTTCAGAGCGAACATGGCACCTGGGTTATCGATAATGAAGGCCGTCGCTATCGCGTGAGCCCGAACT TTCCGATGCGTTTTCATAGCAGCGATGTGATTGTTTTTGGTAGCGATAAAGCGGCCTTTCGCGTGAAAACCATGAAATTTCCGAGCAAAACCGCCGAAGCGAAAGA AGAACGTAAAGTGGTTGGCACCGCGTAAGATCTGGGTACCCTGTTTTGGCGGATGAGAGAAGATTTTCAGCCTGATACAGATTAAATCAGAACGCAGAAGCGGTC TGATAAAACAGAATTTGCCTGGCGGCAGTAGCGCGGTGGTCCCACCTGACCCCATGCCGAACTCAGAAGTGAAACGCCGTAGCGCCGATGGTAGTGTGGGGTCTC CCCATGCGAGAGTAGGGAACTGCCAGGCATCAAATAAAACGAAAGGCTCAGTCGAAAGACTGGGCCTTTCGTTTTATCTGTTGTTTGTCGGTGAACGCTCTCCTGA GTAGGACAAATCCGCCGGGAGCGGATTTGAACGTTGCGAAGCAACGGCCCGGAGGGTGGCGGGCAGGACGCCCGCCATAAACTGCCAGGCATCAAATTAAGCA GAAGGCCATCCTGACGGATGGCCTTTTTGCGTTTCTACAAACTCTTCCTGTCGTCATATCTACAAGCCATCCCCCCACAGATACGGTAAACTAGCCTCGTTTTTGCAT CAGGAAAGCAGCCATGGGCAAATATTATACGCAAGGCGACAAGGTGCTGATGCCGCTGGCGATTCAGGTTCATCATGCCGTCTGTGATGGCTTCCATGTCGGCAG 
AATGCTTAATGAATTACAACAGTACTGCGATGAGTGGCAGGGCGGGGCGTAATTTTTTTAAGGCAGTTATTGGTGCCCTTAAACGCCTGGTGCTACGCCTGAATAA GTGATAATAAGCGGATGAATGGCAGAAATTCGAAAGCAAATTCGACCCGGTCGTCGGTTCAGGGCAGGGTCGTTAAATAGCCGCTTATGTCTATTGCTGGTTTAC CGGTTTATTGACTACCGGAAGCAGTGTGACCGTGTGCTTCTCAAATGCCTGAGGCCAGTTTGCTCAGGCTCTCCCCGTGGAGGTAATAATTGACGATATGATCATTT ATTCTGCCTCCCAGAGCCTGATAAAAACGGTTAGCGCTTCGTTAATACAGATGTAGGTGTTCCACAGGGTAGCCAGCAGCATCCTGCGATGCAGATCCGGAACATA ATGGTGCAGGGCGCTTGTTTCGGCGTGGGTATGGTGGCAGGCCCCGTGGCCGGGGGACTGTTGGGCGCTGCCGGCACCTGTCCTACGAGTTGCATGATAAAGAA GACAGTCATAAGTGCGGCGACGATAGTCATGCCCCGCGCCCACCGGAAGGAGCTACCGGACAGCGGTGCGGACTGTTGTAACTCAGAATAAGAAATGAGGCCGC TCATGGCGTTGACTCTCAGTCATAGTATCGTGGTATCACCGGTTGGTTCCACTCTCTGTTGCGGGCAACTTCAGCAGCACGTAGGGGACTTCCGCGTTTCCAGACTT TACGAAACACGGAAACCGAAGACCATTCATGTTGTTGCTCAGGTCGCAGACGTTTTGCAGCAGCAGTCGCTTCACGTTCGCTCGCGTATCGGTGATTCATTCTGCTA ACCAGTAAGGCAACCCCGCCAGCCTAGCCGGGTCCTCAACGACAGGAGCACGATCATGCGCACCCGTGGCCAGGACCCAACGCTGCCCGAGATGCGCCGCGTGC GGCTGCTGGAGATGGCGGACGCGATGGATATGTTCTGCCAAGGGTTGGTTTGCGCATTCACAGTTCTCCGCAAGAATTGATTGGCTCCAATTCTTGGAGTGGTGA ATCCGTTAGCGAGGTGCCGCCGGCTTCCATTCAGGTCGAGGTGGCCCGGCTCCATGCACCGCGACGCAACGCGGGGAGGCAGACAAGGTATAGGGCGGCGCCTA CAATCCATGCCAACCCGTTCCATGTGCTCGCCGAGGCGGCATAAATCGCCGTGACGATCAGCGGTCCAATGATCGAAGTTAGGCTGGTAAGAGCCGCGAGCGATC CTTGAAGCTGTCCCTGATGGTCGTCATCTACCTGCCTGGACAGCATGGCCTGCAACGCGGGCATCCCGATGCCGCCGGAAGCGAGAAGAATCATAATGGGGAAGG CCATCCAGCCTCGCGTCGCGAACGCCAGCAAGACGTAGCCCAGCGCGTCGGCCGCCATGCCGGCGATAATGGCCTGCTTCTCGCCGAAACGTTTGGTGGCGGGAC CAGTGACGAAGGCTTGAGCGAGGGCGTGCAAGATTCCGAATACCGCAAGCGACAGGCCGATCATCGTCGCGCTCCAGCGAAAGCGGTCCTCGCCGAAAATGACC CAGAGCGCTGCCGGCACCTGTCCTACGAGTTGCATGATAAAGAAGACAGTCATAAGTGCGGCGACGATAGTCATGCCCCGCGCCCACCGGAAGGAGCTGACTGG GTTGAAGGCTCTCAAGGGCATCGGTCGACGCTCTCCCTTATGCGACTCCTGCATTAGGAAGCAGCCCAGTAGTAGGTTGAGGCCGTTGAGCACCGCCGCCGCAAG GAATGGTGCATGCAAGGAGATGGCGCCCAACAGTCCCCCGGCCACGGGGCCTGCCACCATACCCACGCCGAAACAAGCGCTCATGAGCCCGAAGTGGCGAGCCC GATCTTCCCCATCGGTGATGTCGGCGATATAGGCGCCAGCAACCGCACCTGTGGCGCCGGTGATGCCGGCCACGATGCGTCCGGCGTAGAGGATCCACAGGACG GGTGTGGTCGCCATGATCGCGTAGTCGATAGTGGCTCCAAGTAGCGAAGCGAGCAGGACTGGGCGGCGGCCAAAGCGGTCGGACAGTGCTCCGAGAACGGGTG CGCATAGAAATTGCATCAACGCATATAGCGCTAGCAGCACGCCATAGTGACTGGCGATGCTGTCGGAATGGACGATATCCCGCAAGAGGCCCGGCAGTACCGGC ATAACCAAGCCTATGCCTACAGCATCCAGGGTGACGGTGCCGAGGATGACGATGAGCGCATTGTTAGATTTCATACACGGTGCCTGACTGCGTTAGCAATTTAACT GTGATAAACTACCGCATTAAAGCTTATCGATGATAAGCTGTCAAACATGAGAATTACAACTTATATCGTATGGGGCTGACTTCAGGTGCTACATTTGAAGAGATAA ATTGCACTGAAATCTAGAAATATTTTATCTGATTAATAAGATGATCTTCTTGAGATCGTTTTGGTCTGCGCGTAATCTCTTGCTCTGAAAACGAAAAAACCGCCTTGC AGGGCGGTTTTTCGAAGGTTCTCTGAGCTACCAACTCTTTGAACCGAGGTAACTGGCTTGGAGGAGCGCAGTCACCAAAACTTGTCCTTTCAGTTTAGCCTTAACCG GCGCATGACTTCAAGACTAACTCCTCTAAATCAATTACCAGTGGCTGCTGCCAGTGGTGCTTTTTGCATGTCTTTTCGGGTTGGACTCAAGACGATAGTTACCGGATA AGGCGCAGCGGTCGGACTGAACGGGGGGTTCGTGCATACAGTCCAGCTTGGAGCGAACTGCCTACCCGGAACTGAGTGTCAGGCGTGGAATGAGACAAACGCG GCCATAACAGCGGAATGACACCGGTAAACCGAAAGGCAGGAACAGGAGAGCGCACGAGGGAGCCGCCAGGGGGAAACGCCTGGTATCTTTATAGTCCTGTCGG GTTTCGCCACCACTGATTTGAGCGTCAGATTTCGTGATGCTTGTCAGGGGGGCGGAGCCTATGGAAAAACGGCTTTGCCGCGGCCCTCTCACTTCCCTGTTAAGTA TCTTCCTGGCATCTTCCAGGAAATCTCCGCCCCGTTCGTAAGCCATTTCCGCTCGCCGCAGTCGAACGACCGAGCGTAGCGAGTCAGTGAGCGAGGAAGCGGAAT ATATCCTGTATCACATATTCTGCTGACGCACCGGTGCAGCCTTTTTTTCTCCTGCCACATGAAGCACTTCACTGACACCCTCATCAGTGCCAACATAGTAAGCCAGTAT ACACTCCGCTAGCGCTGATGTCCGGCGGTGCTTTTGCCGTTACGCACCACCCCGTCAGTAGCTGAACAGGAGGGACAGCTGATAGAAACAGAAGCCACTGGAGCA CCTCAAAAACACCATCATACACTAAATCAGTAAGTTGGCAGCATCACCCGACGCACTTTGGGCCGAATAAATACCTGTGACGGAAGATCACTTCGCAGAATAAATA AATCCTGGTGTCCCTGTTGATACCGGGAAGCCCTGGGCCAACTTTTGGCGAAAATGAGACGTTGATCGGCACGTAAGAGGTTCCAACTTTCACCATAATGAAATAA GATCACTACCGGGCGTATTTTTTGAGTTATCGAGATTTTCAGGAGCTAAGGAAGCTAAAATGGAGAAAAAAATCACTGGATATACCACCGTTGATATATCCCAATG GCATCGTAAAGAACATTTTGAGGCATTTCAGTCAGTTGCTCAATGTACCTATAACCAGACCGTTCAGCTGGATATTACGGCCTTTTTAAAGACCGTAAAGAAAAATA AGCACAAGTTTTATCCGGCCTTTATTCACATTCTTGCCCGCCTGATGAATGCTCATCCGGAATTC

pAClac-viol-2 AGCTGTTGACAATTAATCATCGGCTCGTATAATGTGTGGAATTGTGAGCGGATAACAATTTCACACAGGAAACACTCGAGGAGGCAGCTATGCTTCGTTCGTTGCT CAGAGGCCTCACGCATTTCCCCCGCGTGAACTCCGCGCAGCAGCCCAGCTGTGCACACGCGCGACTCCAGTTTAGGCCCAGAAGCATGCAGCTGCTTGCCGAGGA CCGCACAGACCATATGAGGGGTGCAAGTACCTGGGCAGGCGGGCAGTCGCAGGATGAGCTGATGCTGAAGGACGAGTGCATCTTGGTGGATGCTGACGACAAC ATTACAGGCCATGTCAGCAAGCTGGAGTGCCACAAGTTCCTACCACATCAGCCTGCAGGCCTGCTGCACCGGGCCTTCTCTGTATTCCTGTTTGACGACCAGGGGC GACTGCTGCTGCAACAGCGTGCACGATCAAAAATCACATTCCCCAGTGTGTGGACCAACACCTGCTGCAGCCACCCTCTACATGGGCAGACCCCAGATGAGGTGG ACCAACTAAGCCAGGTGGCCGACGGCACAGTACCTGGCGCAAAGGCTGCTGCCATCCGCAAGTTGGAGCACGAGCTGGGGATACCAGCGCACCAGCTGCCGGCC AGCGCGTTTCGCTTCCTCACGCGTTTGCACTACTGCGCCGCGGACGTGCAGCCGGCTGCGACACAATCAGCACTCTGGGGCGAGCACGAAATGGACTACATCTTAT TCATCCGGGCCAACGTCACCCTTGCGCCCAACCCTGACGAGGTGGACGAAGTCAGGTACGTGACGCAGGAGGAGCTGCGGCAGATGATGCAGCCGGACAATGGG TTGCAATGGTCGCCGTGGTTTCGCATCATCGCCGCGCGCTTCCTTGAGCGCTGGTGGGCTGACCTAGACGCGGCCCTGAACACTGACAAACACGAGGATTGGGGA ACGGTGCATCACATCAACGAAGCGTGAGATCCAGGAGGCAGCTATGACGGTCTGCGCAAAAAAACACGTTCATCTCACTCGCGATGCTGCGGAGCAGTTACTGGC TGATATTGATCGACGCCTTGATCAGTTATTGCCCGTGGAGGGAGAACGGGATGTTGTGGGTGCCGCGATGCGTGAAGGTGCGCTGGCACCGGGAAAACGTATTC GCCCCATGTTGCTGTTGCTGACCGCCCGCGATCTGGGTTGCGCTGTCAGCCATGACGGATTACTGGATTTGGCCTGTGCGGTGGAAATGGTCCACGCGGCTTCGC GATCCTTGACGATATGCCCTGCATGGACGATGCGAAGCTGCGGCGCGGACGCCCTACCATTCATTCTCATTACGGAGAGCATGTGGCAATACTGGCGGCGGTTGC CTTGCTGAGTAAAGCCTTTGGCGTAATTGCCGATGCAGATGGCCTCACGCCGCTGGCAAAAAATCGGGCGGTTTCTGAACTGTCAAACGCCATCGGCATGCAAGG ATTGGTTCAGGGTCAGTTCAAGGATCTGTCTGAAGGGGATAAGCCGCGCAGCGCTGAAGCTATTTTGATGACGAATCACTTTAAAACCAGCACGCTGTTTTGTGCC TCCATGCAGATGGCCTCGATTGTTGCGAATGCCTCCAGCGAAGCGCGTGATTGCCTGCATCGTTTTTCACTTGATCTTGGTCAGGCATTTCAACTGCTGGACGATTT GACCGATGGCATGACCGACACCGGTAAGGATAGCAATCAGGACGCCGGTAAATCGACGCTGGTCAATCTGTTAGGCCCGAGGGCGGTTGAAGAACGTCTGAGAC AACATCTTCAGCTTGCCAGTGAGCATCTCTCTGCGGCCTGCCAACACGGGCACGCCACTCAACATTTTATTCAGGCCTGGTTTGACAAAAAACTCGCTGCCGTCAGT TAAATCTAGAAGATCCAGGAGGCAGCTATGAATAATCCGTCGTTACTCAATCATGCGGTCGAAACGATGGCAGTTGGCTCGAAAAGTTTTGCGACAGCCTCAAAG TATTTGATGCAAAAACCCGGCGCAGCGTACTGATGCTCTACGCCTGGTGCCGCCATTGTGACGATGTTATTGACGATCAGACGCTGGGCTTTCAGGCCCGGCAGCC TGCCTTACAAACGCCCGAACAACGTCTGATGCAACTTGAGATGAAAACGCGCCAGGCCTATGCAGGATCGCAGATGCACGAACCGGCGTTTGCGGCTTTTCAGGA AGTGGCTATGGCTCATGATATCGCCCCGGCTTACGCGTTTGATCATCTGGAAGGCTTCGCCATGGATGTACGCGAAGCGCAATACAGCCAACTGGATGATACGCTG CGCTATTGCTATCACGTTGCAGGCGTTGTCGGCTTGATGATGGCGCAAATCATGGGCGTGCGGGATAACGCCACGCTGGACCGCGCCTGTGACCTTGGGCTGGCA TTTCAGTTGACCAATATTGCTCGCGATATTGTGGACGATGCGCATGCGGGCCGCTGTTATCTGCCGGCAAGCTGGCTGGAGCATGAAGGTCTGAACAAAGAGAAT TATGCGGCACCTGAAAACCGTCAGGCGCTGAGCCGTATCGCCCGTCGTTTGGTGCAGGAAGCAGAACCTTACTATTTGTCTGCCACAGCCGGCCTGGCAGGGTTG CCCCTGCGTTCCGCCTGGGCAATCGCTACGGCGAAGCAGGTTTACCGGAAAATAGGTGTCAAAGTTGAACAGGCCGGTCAGCAAGCCTGGGATCAGCGGCAGTC AACGACCACGCCCGAAAAATTAACGCTGCTGCTGGCCGCCTCTGGTCAGGCCCTTACTTCCCGGATGCGGGCTCATCCTCCCCGCCCTGCGCATCTCTGGCAGCGC CCGCTCTAGCAATTCAGGAGGCAGCTATGAAACCAACTACGGTAATTGGTGCAGGCTTCGGTGGCCTGGCACTGGCAATTCGTCTACAAGCTGCGGGGATCCCCG TCTTACTGCTTGAACAACGTGATAAACCCGGCGGTCGGGCTTATGTCTACGAGGATCAGGGGTTTACCTTTGATGCAGGCCCGACGGTTATCACCGATCCCAGTGC CATTGAAGAACTGTTTGCACTGGCAGGAAAACAGTTAAAAGAGTATGTCGAACTGCTGCCGGTTACGCCGTTTTACCGCCTGTGTTGGGAGTCAGGGAAGGTCTTT AATTACGATAACGATCAAACCCGGCTCGAAGCGCAGATTCAGCAGTTTAATCCCCGCGATGTCGAAGGTTATCGTCAGTTTCTGGACTATTCACGCGCGGTGTTTA AAGAAGGCTATCTAAAGCTCGGTACTGTCCCTTTITTATCGTTCAGAGACATGCTTCGCGCCGCACCTCAACTGGCGAAACTGCAGGCATGGAGAAGCGTTTACAG TAAGGTTGCCAGTTACATCGAAGATGAACATCTGCGCCAGGCGTTTTCTTTCCACTCGCTGTTGGTGGGCGGCAATCCCTTCGCCACCTCATCCATTTATACGTTGAT ACACGCGCTGGAGCGTGAGTGGGGCGTCTGGTTTCCGCGTGGCGGCACCGGCGCATTAGTTCAGGGGATGATAAAGCTGTTTCAGGATCTGGGTGGCGAAGTCG TGTTAAACGCCAGAGTCAGCCATATGGAAACGACAGGAAACAAGATTGAAGCCGTGCATTTAGAGGACGGTCGCAGGTTCCTGACGCAAGCCGTCGCGTCAAAT GCAGATGTGGTTCATACCTATCGCGACCTGTTAAGCCAGCACCCTGCCGCGGTTAAGCAGTCCAACAAACTGCAGACTAAGCGCATGAGTAACTCTCTGTTTGTGC TCTATTTTGGTTTGAATCACCATCATGATCAGCTCGCGCATCACACGGTTTGTTTCGGCCCGCGTTACCGCGAGCTGATTGACGAAATTTTTAATCATGATGGCCTCG CAGAGGACTTCTCACTTTATCTGCACGCGCCCTGTGTCACGGATTCGTCACTGGCGCCTGAAGGTTGCGGCAGTTACTATGTGTTGGCGCCGGTGCCGCATTTAGG CACCGCGAACCTCGACTGGACGGTTGAGGGGCCAAAACTACGCGACCGTATTTTTTGCGTACCTTGAGCAGCATTACATGCCTGGCTTACGGAGTCAGCTGGTCAC GCACCGGATGTTTACGCCGTTTGATTTTCGCGACCAGCTTAATGCCTATCATGGCTCAGCCTTTTCTGTGGAGCCCGTTCTTACCCAGAGCGCCTGGTTTCGGCCGC ATAACCGCGATAAAACCATTACTAATCTCTACCTGGTCGGCGCAGGCACGCATCCCGGCGCAGGCATTCCTGGCGTCATCGGCTCGGCAAAAGCGACAGCAGGTT GATGCTGGAGGATCTGATATGAGATCCAGGAGGCAGCTATGCAACCGCATTATGATCTGATTCTCGTGGGGGCTGGACTCGCGAATGGCCTTATCGCCCTGCGTC TTCAGCAGCAGCAACCTGATATGCGTATTTTGCTTATCGACGCCGCACCCCAGGCGGGCGGGAATCATACGTGGTCATTTCACCACGATGATTTGACTGAGAGCCA ACATCGTTGGATAGCTCCGCTGGTGGTTCATCACTGGCCCGACTATCAGGTACGCTTTCCCACACGCCGTCGTAAGCTGAACAGCGGCTACTTTTGTATTACTTCTC AGCGTTTCGCTGAGGTTTTACAGCGACAGTTTGGCCCGCACTTGTGGATGGATACCGCGGTCGCAGAGGTTAATGCGGAATCTGTTCGGTTGAAAAAGGGTCAGG TTATCGGTGCCCGCGCGGTGATTGACGGGCGGGGTTATGCGGCAAATTCAGCACTGAGCGTGGGCTTCCAGGCGTTTATTGGCCAGGAATGGCGATTGAGCCACC CGCATGGTTTATCGTCTCCCATTATCATGGATGCCACGGTCGATCAGCAAAATGGTTATCGCTTCGTGTACAGCCTGCCGCTCTCGCCGACCAGATTGTTAATTGAA GACACGCACTATATTGATAATGCGACATTAGATCCTGAATGCGCGCGGCAAAATATTTGCGACTATGCCGCGCAACAGGGTTGGCAGCTTCAGACACTGCTGCGA GAAGAACAGGGCGCCTTACCCATTACTCTGTCGGGCAATGCCGACGCATTCTGGCAGCAGCGCCCCCTGGCCTGTAGTGGATTACGTGCCGGTCTGTTCCATCCTA 
CCACCGGCTATTCACTGCCGCTGGCGGTTGCCGTGGCCGACCGCCTGAGTGCACTTGATGTCTTTTACGTCGGCCTCAATTCACCATGCCATTACGCATTTTGCCCGC GAGCGCTGGCAGCAGCAGGGCTTTTTCCGCATGCTGAATCGCATGCTGTTTTTAGCCGGACCCGCCGATTCACGCTGGCGGGTTATGCAGCGTTTTTATGGTTTAC CTGAAGATTTAATTGCCCGTTTTTATGCGGGAAAACTCACGCTGACCGATCGGCTACGTATTCTGAGCGGCAAGCCGCCTGTTCCGGTATTAGCAGCATTGCAAGC CATTATGACGACTCATCGTTAAGGTACCAGCTGTTGACAATTAATCATCGGCTCGTATAATGTGTGGAATTGTGAGCGGATAACAATTTCACACGATCCAGGAGGC AGCTATGTTGTGGATTTGGAATGCCCTGATCGTTTTCGTTACCGTGATTGGCATGGAAGTGATTGCTGCACTGGCACACAAATACATCATGCACGGCTGGGGTTGG GGATGGCATCTTTCACATCATGAACCGCGTAAAGGTGCGTTTGAAGTTAACGATCTTTATGCCGTGGTTTTTGCTGCATTATCGATCCTGCTGATTTATCTGGGCAG TACAGGAATGTGGCCGCTCCAGTGGATTGGCGCAGGTATGACGGCGTATGGATTACTCTATTTTATGGTGCACGACGGGCTGGTGCATCAACGTTGGCCATTCCG CTATATTCCACGCAAGGGCTACCTCAAACGGTTGTATATGGCGCACCGTATGCATCACGCCGTCAGGGGCAAAGAAGGTTGTGTTTCTTTTGGCTTCCTCTATGCGC CGCCCCTGTCAAAACTTCAGGCGACGCTCCGGGAAAGACATGGCGCTAGAGCGGGCGCTGCCAGAGATGCGCAGGGCGGGGAGGATGAGCCCGCATCCGGGAA GTAAGATCCAGGAACTAGTTGATTTAATATAAGGAGGTAGAAAAATGACCCTGGCCGAAGCACCGGCCACCCCGACCGAAAAATCTAACAGCGAAGTGCCGCAG AAAAAACTGAAAGTGCTGGTTGCCGGCGGTGGCATTGGTGGCCTGGTGTTTTGCGCTGGCGGCGAAAAAGAAAGGCTTTGATGTGCTGGTTTTTGAACGTGATCTG AGCGCCATCCGCGGCGAAGGCCAGTATCGTGGCCCGATTCAGATCCAGAGCAATGCCCTGGCGGCCCTGGAAGCGATTGATATGGATGTGGCCGAAGAAATCAT GAACGCGGGTTGCATTACCGGCCAGCGTATCAATGGTCTGGTTGATGGTATTAGCGGCAACTGGTATTGTAAATTTGATACCTTTACCCCGGCCGTGGAACGCGGC CTGCCGGTGACCCGTGTTATTAGCCGCATGACCCTGCAGCAGATCCTGGCACGTGCCGTGGGTGAAGATGTTATTATGAACGAAAGCAATGTGGTTAACTTTGAA GATGATGGCGAAAAAGTGACCGTGGTTCTGGAAAATGGTCAGCGCTTTACCGGCGATCTGCTGGTTGGTGCCGATGGCATCCGTAGCAAAGTGCGCACCAACCTG TTTGGTCATAGCGAAGCGACCTATAGCGGTTATACCTGTTATACCGGCATTGCCGATTTTTGTTCCGGCGGATATCGATACCGTGGGTTATCGTGTTTTTCTGGGCCA TAAACAGTATTTTGTGAGCAGCGATGTTGGTGGCGGTAAAATGCAGTGGTATGCGTTTCATAATGAACCGGCCGGCGGTGTGGATGCCCCGAATGGCAAGAAAG AACGCCTGCTGAAAATTTTTGGCGGTTGGTGCGATAATGTGATCGATCTGCTGGTTGCCACCGATGAAGATGCGATTCTGCGTCGCGATATCTATGATCGTCCGCC GACCTTTAATTGGGGTCGTGGTCGTGTGACCCTGCTGGGTGATAGCGTTCATGCCATGCAGCCGAACCTGGGTCAGGGCGGTTGTATGGCCATTGAAGATAGCTA TCAGCTGGCGCTGGAACTGGAAAAAGCCTGGAGCCGCAGCGCCGAAAGCGGTAGCCCGATGGATGTTATCAGCAGCCTGCGTAGCTATGAAAGCGCCCGTAAAC TGCGCGTGGGTGTTATTCATGGCCTGGCCCGTATGGCCGCAATCATGGCAAGCACCTATAAAGCGTATCTGGGCGTGGGTCTGGGTCCGCTGAGCTTTCTGACCAA ATATCGTATTCCGCACCCGGGTCGTGTTGGCGGTCGTGTGTTTGTTGATCTGGGCATGCCGCTGATGCTGAGCTGGGTGCTGGGCGGTAATGGTGATAAACTGGA AGGCCGTATCCAGCATTGCCGCCTGAGCGAAAAAGCCAACGATCAGCTGCGTCGCTGGTTTGAAGATGATGATGCGCTGGAACGCGCCACCGATGCCGAATGGC GCTGCTGCCGGCCGCAAATGGCAATAGCGCGCTGGAAACCATTGTGCTGAGCCGTGATGAAGATGTTCCGTGCACCATTGGTAGCGTGAGCCATACCAACATCCC GGGCAAAAGCGTGGTTCTGCCGCTGCCGCAGGTTAGCGAAATGCATGCCCAGATTAGCTGTAAAAACAATGCGTTTTTCGTGACCGATTTTCAGAGCGAACATGG TACCTGGGTTATCGATAATGAAGGCCGTCGCTATCGCGTGAGCCCGAACTTTCCGATGCGTTTTCATAGCAGCGATGTGATTGTTTTTGGTAGCGATAAAGCGGCC TTTCGCGTGAAAACCATGAAATTTCCGAGCAAAACCGCCGAAGCGAAAGAAGAACGTAAAGTGGTTGGCACCGCGTAAAAGCTTGTACCCTGTTTTGGCGGATGA GAGAAGATTTTCAGCCTGATACAGATTAAATCAGAACGCAGAAGCGGTCTGATAAAACAGAATTTGCCTGGCGGCAGTAGCGCGGTGGTCCCACCTGACCCCATG CCGAACTCAGAAGTGAAACGCCGTAGCGCCGATGGTAGTGTGGGGTCTCCCCATGCGAGAGTAGGGAACTGCCAGGCATCAAATAAAACGAAAGGCTCAGTCGA AAGACTGGGCCTTTCGTTTTATCTGTTGTTTGTCGGTGAACGCTCTCCTGAGTAGGACAAATCCGCCGGGAGCGGATTTGAACGTTGCGAAGCAACGGCCCGGAG GGTGGCGGGCAGGACGCCCGCCATAAACTGCCAGGCATCAAATTAAGCAGAAGGCCATCCTGACGGATGGCCTTTTTGCGTTTTGCCTAATGAGTGAACTCACATT AATTGCGTTGCGCTCACTGCCCGCTTTCCAGTCGGGAAACCTGTCGTGCCAGCTGCATTAATGAATCGGCCAACGCGCGGGGAGAGGCGGTTTGCGTATTGGGCG CCAGGGTGGTTTTTCTTTTCACCAGTGAGACGGGCAACAGCTGATTGCCCTTCACCGCCTGGCCCTGAGAGAGTTGCAGCAAGCGGTCCACGCTGGTTTGCCCCAG CAGGCGAAAATCCTGTTTGATGGTGGTTAACGGCGGGATATAACATGAGCTGTCTTCGGTATCGTCGTATCCCACTACCGAGATATCCGCACCAACGCGCAGCCCG GACTCGGTAATGGCGCGCATTGCGCCCAGCGCCATCTGATCGTTGGCAACCAGCATCGCAGTGGGAACGATGCCCTCATTCAGCATTTGCATGGTTTGTTGAAAAC CGGACATGGCACTCCAGTCGCCTTCCCGTTCCGCTATCGGCTGAATTTGATTGCGAGTGAGATATTTATGCCAGCCAGCCAGACGCAGACGCGCCGAGACAGAACT TAATGGGCCCGCTAACAGCGCGATTTGCTGGTGACCCAATGCGACCAGATGCTCCACGCCCAGTCGCGTACCGTCTTCATGGGAGAAAATAATACTGTTGATGGG GTCTGGTCAGAGACATCAAGAAATAACGCCGGAACATTAGTGCAGGCAGCTTCCACAGCAATGGCATCCTGGTCATCCAGCGGATAGTTAATGATCAGCCCACTG ACGCGTTGCGCGAGAAGATTGTGCACCGCCGCTTTACAGGCTTCGACGCCGCTTCGTTCTACCATCGACACCACCACGCTGGCACCCAGTTGATCGGCGCGAGATT TAATCGCCGCGACAATTTGCGACGGCGCGTGCAGGGCCAGACTGGAGGTGGCAACGCCAATCAGCAACGACTGTTTGCCCGCCAGTTGTTGTGCCACGCGGTTGG GAATGTAATTCAGCTCCGCCATCGCCGCTTCCACTTTTTCCCGCGTTTTCGCAGAAACGTGGCTGGCCTGGTTCACCACGCGGGAAACGGTCTGATAAGAGACACC GGCATACTCTGCGACATCGTATAACGTTACTGGTTTCACATTCACCACCCTGAATTGACTCTCTTCCGGGCGCTATCATGCCATACCGCGAAAGGTTTTGCACCATTC GATGGGGCGTAGCACCAGGCGTTTAAGGGCACCAATAACTGCCTTAAAAAAATTACGCCCCGCCCTGCCACTCATCGCAGTACTGTTGTAATTCATTAAGCATTCTG CCGACATGGAAGCCATCACAGACGGCATGATGAACCTGAATCGCCAGCGGCATCAGCACCTTGTCGCCTTGCGTATAATATTTGCCCATGGTGAAAACGGGGGCG AAGAAGTTGTCCATATTGGCCACGTTTAAATCAAAACTGGTGAAACTCACCCAGGGATTGGCTGAGACGAAAAACATATTCTCAATAAACCCTTTAGGGAAATAGG CCAGGTTTTCACCGTAACACGCCACATCTTGCGAATATATGTGTAGAAACTGCCGGAAATCGTCGTGGTATTCACTCCAGAGCGATGAAAACGTTTCAGTTTGCTCA TGGAAAACGGTGTAACAAGGGTGAACACTATCCCATATCACCAGCTCACCGTCTTTCATTGCCATACGGAATTCCGGATGAGCATTCATCAGGCGGGCAAGAATGT GAATAAAGGCCGGATAAAACTTGTGCTTATTTTTCTTTACGGTCTTTAAAAAGGCCGTAATATCCAGCTGAACGGTCTGGTTATAGGTACATTGAGCAACTGACTGA AATGCCTCAAAATGTTCTTTACGATGCCATTGGGATATATCAACGGTGGTATATCCAGTGATTTTTTTTCTCCATTTTAGCTTCCTTAGCTCCTGAAAATCTCGATAACT CAAAAAATACGCCCGGTAGTGATCTTATTTCATTATGGTGAAAGTTGGAACCTCTTACGTGCCGATCAACGTCTCATTTTCGCCAAAAGTTGGCCCAGGGCTTCCCG GTATCAACAGGGACACCAGGATTTATTTATTCTGCGAAGTGATCTTCCGTCACAGGTATTTATTCGGCGCAAAGTGCGTCGAGCTTATCGATGATAAGCTGTCAAAC ATGAGAATTACAACTTATATCGTATGGGGCTGACTTCAGGTGCTACATTTGAAGAGATAAATTGCACTGAAATCTAGAAATATTTTATCTGATTAATAAGATGATCT TCTTGAGATCGTTTTTGGTCTGCGCGTAATCTCTTGCTCTGAAAACGAAAAAACCGCCTTGCAGGGCGGTTTTTCGAAGGTTCTCTGAGCTACCAACTCTTTGAACCG AGGTAACTGGCTTGGAGGAGCGCAGTCACCAAAACTTGTCCTTTCAGTTTAGCCTTAACCGGCGCATGACTTCAAGACTAACTCCTCTAAATCAATTACCAGTGGCT GCTGCCAGTGGTGCTTTTGCATGTCTTTCCGGGTTGGACTCAAGACGATAGTTACCGGATAAGGCGCAGCGGTCGGACTGAACGGGGGGTTCGTGCATACAGTCC AGCTTGGAGCGAACTGCCTACCCGGAACTGAGTGTCAGGCGTGGAATGAGACAAACGCGGCCATAACAGCGGAATGACACCGGTAAACCGAAAGGCAGGAACA GGAGAGCGCACGAGGGAGCCGCCAGGGGGAAACGCCTGGTATCTTTATAGTCCTGTCGGGTTTCGCCACCACTGATTTGAGCGTCAGATTTCGTGATGCTTGTCA GGGGGGCGGAGCCTATGGAAAAACGGCTTTGCCGCGGCCCTCTCACTTCCCTGTTAAGTATCTTCCTGGCATCTTCCAGGAAATCTCCGCCCCGTTCGTAAGCCAT TCCGCTCGCCGCAGTCGAACGACCGAGCGTAGCGAGTCAGTGAGCGAGGAAGCGGAATATATCCTGTATCACATATTCTGCTGACGCACCGGTGCAGCCTTTTTTC TCCTGCCACATGAAGCACTTCACTGACACCCTCATCAGTGCCAACATAGTAAGCCAGTATACACTCCGCTAGCGCTGATGTCCGGCGGTGCTTTTGCCGTTACGCAC CACCCCGTCAGTAGCTGAACAGGAGGGACAGCTGATAGAAACAGAAGCCACTGGAGCACCTCAAAAACACCATCATACACTAAATCAGTAAGTTGGCAGCATCAC CCGACGCACTTTGCGCCGAATAAATACCTGTGACGGAAGATCACTTCGCAGAATAAATAAATCCTGGTGTCCCTGTTGATACCGGGAAGCCCTGGGCCAACTTTTG GCGAAAATGAGACGTTGATCGGCACGTAAGAGGTTCCAACTTTCACCATAATGAAATAAGATCACTACCGGGCGTATTTTTTGAGTTATCGAGATTTTCAGGAGCT AAGGAAGCTAAAATGGAGAAAAAAATCACTGGATATACCACCGTTGATATATCCCAATGGCATCGTAAAGAACATTTTGAGGCATTTCAGTCAGTTGCTCAATGTA CCTATAACCAGACCGTTCAGCTGGATATTACGGCCTTTTTAAAGACCGTAAAGAAAAATAAGCACAAGTTTTATCCGGCCTTTATTCACATTCTTGCCCGCCTGATGA ATGCTCATCCGGAATTC 
Table S4. DNA sequences of constructs based on pUC18, pUC57 or pUCara vectors.

\begin{tabular}{|c|c|}
\hline Plasmid Name & equence \\
\hline $\begin{array}{l}\text { pUC18-CaCCS } \\
\text { (Vector: } \\
\text { pUC18(commercial } \\
\text { Cloning site: } \\
\text { EcoRI/HindIII) }\end{array}$ & 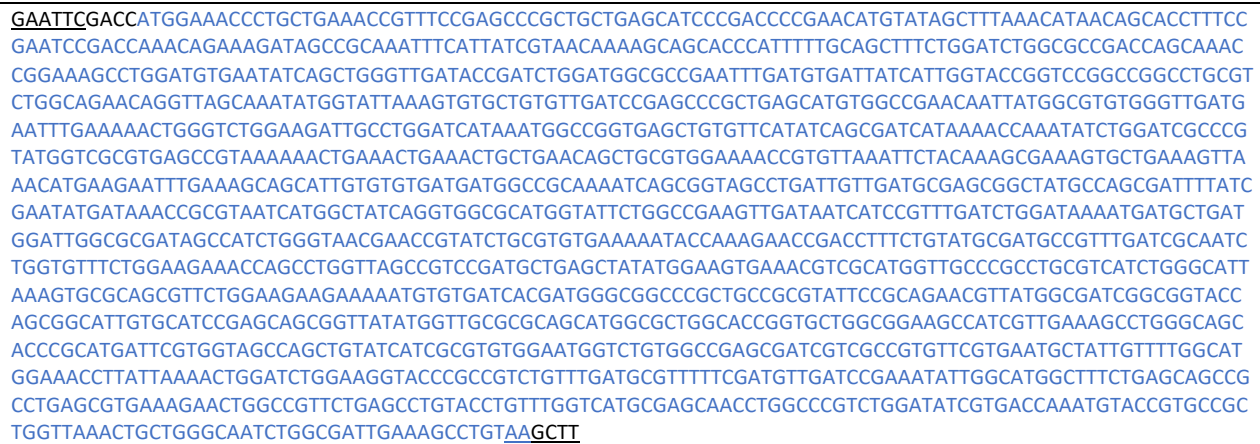 \\
\hline
\end{tabular}

pUC57-CaZEP

(Vector: pUC57-

Kan(commercial),

Cloning site:

HindIII/BamHI)

AAGCTTGATGTATAGCACCGTGTTTTATACCAGCGTTCATCCGAGCACCAGCGTGTTTAGCCGCAAACAGCTGCCGCTGCTGATTAGCAAAGATTTTCC GGCCGAACTGTATCATAGCCTGCCGTGCAAAAGCCTGGAAAATGGTCATATTAAGAAAGTGAAAGGCGTTAAAGCCACCCTGGCCGAAGCACCGGCC ACCCCGACCGAAAAATCTAACAGCGAAGTGCCGCAGAAAAAACTGAAAGTGCTGGTTGCCGGCGGTGGCATTGGTGGCCTGGTGTTTGCGCTGGCG GCGAAAAAGAAAGGCTTTGATGTGCTGGTTTTTGAACGTGATCTGAGCGCCATCCGCGGCGAAGGCCAGTATCGTGGCCCGATTCAGATCCAGAGCA ATGCCCTGGCGGCCCTGGAAGCGATTGATATGGATGTGGCCGAAGAAATCATGAACGCGGGTTGCATTACCGGCCAGCGTATCAATGGTCTGGTTGA TGGTATTAGCGGCAACTGGTATTGTAAATTTGATACCTTTACCCCGGCCGTGGAACGCGGCCTGCCGGTGACCCGTGTTATTAGCCGCATGACCCTGC AGCAGATCCTGGCACGTGCCGTGGGTGAAGATGTTATTATGAACGAAAGCAATGTGGTTAACTTTGAAGATGATGGCGAAAAAGTGACCGTGGTTCT GGAAAATGGTCAGCGCTTTACCGGCGATCTGCTGGTTGGTGCCGATGGCATCCGTAGCAAAGTGCGCACCAACCTGTTTGGTCATAGCGAAGCGACC TATAGCGGTTATACCTGTTATACCGGCATTGCCGATTTTGTTCCGGCGGATATCGATACCGTGGGTTATCGTGTTTTTCTGGGCCATAAACAGTATTTTG TGAGCAGCGATGTTGGTGGCGGTAAAATGCAGTGGTATGCGTTTCATAATGAACCGGCCGGCGGTGTGGATGCCCCGAATGGCAAGAAAGAACGCC TGCTGAAAATTTTTGGCGGTTGGTGCGATAATGTGATCGATCTGCTGGTTGCCACCGATGAAGATGCGATTCTGCGTCGCGATATCTATGATCGTCCGC CGACCTTTAATTGGGGTCGTGGTCGTGTGACCCTGCTGGGTGATAGCGTTCATGCCATGCAGCCGAACCTGGGTCAGGGCGGTTGTATGGCCATTGA AGATAGCTATCAGCTGGCGCTGGAACTGGAAAAAGCCTGGAGCCGCAGCGCCGAAAGCGGTAGCCCGATGGATGTTATCAGCAGCCTGCGTAGCTA TGAAAGCGCCCGTAAACTGCGCGTGGGTGTTATTCATGGCCTGGCCCGTATGGCCGCAATCATGGCAAGCACCTATAAAGCGTATCTGGGCGTGGGT CTGGGTCCGCTGAGCTTTCTGACCAAATATCGTATTCCGCACCCGGGTCGTGTTGGCGGTCGTGTGTTTGTTGATCTGGGCATGCCGCTGATGCTGAG CTGGGTGCTGGGCGGTAATGGTGATAAACTGGAAGGCCGTATCCAGCATTGCCGCCTGAGCGAAAAAGCCAACGATCAGCTGCGTCGCTGGTTTGAA GATGATGATGCGCTGGAACGCGCCACCGATGCCGAATGGCTGCTGCTGCCGGCCGCAAATGGCAATAGCGCGCTGGAAACCATTGTGCTGAGCCGT GATGAAGATGTTCCGTGCACCATTGGTAGCGTGAGCCATACCAACATCCCGGGCAAAAGCGTGGTTCTGCCGCTGCCGCAGGTTAGCGAAATGCATG CCCAGATTAGCTGTAAAAACAATGCGTTTTTCGTGACCGATTTTCAGAGCGAACATGGTACCTGGGTTATCGATAATGAAGGCCGTCGCTATCGCGTG AGCCCGAACTTTCCGATGCGTTTTCATAGCAGCGATGTGATTGTTTTTGGTAGCGATAAAGCGGCCTTTCGCGTGAAAACCATGAAATTTCCGAGCAAA ACCGCCGAAGCGAAAGAAGAACGTAAAGTGGTTGGCACCGCGTAAGGAAAGGAACCATGGATCC

pUC57-CaZEP-CaCCS (Vector: pUC57Kan(commercial), Cloning site: HindIII/BamHI)
pUCara-CaCCS ${ }_{\mathrm{M} 40}$
AAGCTTGATGTATAGCACCGTGTTTTATACCAGCGTTCATCCGAGCACCAGCGTGTTTAGCCGCAAACAGCTGCCGCTGCTGATTAGCAAAGATTTTCC GGCCGAACTGTATCATAGCCTGCCGTGCAAAAGCCTGGAAAATGGTCATATTAAGAAAGTGAAAGGCGTTAAAGCCACCCTGGCCGAAGCACCGGCC ACCCCGACCGAAAAATCTAACAGCGAAGTGCCGCAGAAAAAACTGAAAGTGCTGGTTGCCGGCGGTGGCATTGGTGGCCTGGTGTTTGCGCTGGCG GCGAAAAAGAAAGGCTTTGATGTGCTGGTTTTTGAACGTGATCTGAGCGCCATCCGCGGCGAAGGCCAGTATCGTGGCCCGATTCAGATCCAGAGCA ATGCCCTGGCGGCCCTGGAAGCGATTGATATGGATGTGGCCGAAGAAATCATGAACGCGGGTTGCATTACCGGCCAGCGTATCAATGGTCTGGTTGA TGGTATTAGCGGCAACTGGTATTGTAAATTTGATACCTTTACCCCGGCCGTGGAACGCGGCCTGCCGGTGACCCGTGTTATTAGCCGCATGACCCTGC AGCAGATCCTGGCACGTGCCGTGGGTGAAGATGTTATTATGAACGAAAGCAATGTGGTTAACTTTGAAGATGATGGCGAAAAAGTGACCGTGGTTCT GGAAAATGGTCAGCGCTTTACCGGCGATCTGCTGGTTGGTGCCGATGGCATCCGTAGCAAAGTGCGCACCAACCTGTTTGGTCATAGCGAAGCGACC TATAGCGGTTATACCTGTTATACCGGCATTGCCGATTTTTGTTCCGGCGGATATCGATACCGTGGGTTATCGTGTTTTTCTGGGCCATAAACAGTATTTTTG TGAGCAGCGATGTTGGTGGCGGTAAAATGCAGTGGTATGCGTTTCATAATGAACCGGCCGGCGGTGTGGATGCCCCGAATGGCAAGAAAGAACGCC TGCTGAAAATTTTTGGCGGTTGGTGCGATAATGTGATCGATCTGCTGGTTGCCACCGATGAAGATGCGATTCTGCGTCGCGATATCTATGATCGTCCGC CGACCTTTAATTGGGGTCGTGGTCGTGTGACCCTGCTGGGTGATAGCGTTCATGCCATGCAGCCGAACCTGGGTCAGGGCGGTTGTATGGCCATTGA AGATAGCTATCAGCTGGCGCTGGAACTGGAAAAAGCCTGGAGCCGCAGCGCCGAAAGCGGTAGCCCGATGGATGTTATCAGCAGCCTGCGTAGCTA TGAAAGCGCCCGTAAACTGCGCGTGGGTGTTATTCATGGCCTGGCCCGTATGGCCGCAATCATGGCAAGCACCTATAAAGCGTATCTGGGCGTGGGT CTGGGTCCGCTGAGCTTTCTGACCAAATATCGTATTCCGCACCCGGGTCGTGTTGGCGGTCGTGTGTTTGTTGATCTGGGCATGCCGCTGATGCTGAG CTGGGTGCTGGGCGGTAATGGTGATAAACTGGAAGGCCGTATCCAGCATTGCCGCCTGAGCGAAAAAGCCAACGATCAGCTGCGTCGCTGGTTTGAA GATGATGATGCGCTGGAACGCGCCACCGATGCCGAATGGCTGCTGCTGCCGGCCGCAAATGGCAATAGCGCGCTGGAAACCATTGTGCTGAGCCGT GATGAAGATGTTCCGTGCACCATTGGTAGCGTGAGCCATACCAACATCCCGGGCAAAAGCGTGGTTCTGCCGCTGCCGCAGGTTAGCGAAATGCATG CCCAGATTAGCTGTAAAAACAATGCGTTTTTCGTGACCGATTTTCAGAGCGAACATGGTACCTGGGTTATCGATAATGAAGGCCGTCGCTATCGCGTG AGCCCGAACTTTCCGATGCGTTTTCATAGCAGCGATGTGATTGTTTTTGGTAGCGATAAAGCGGCCTTTCGCGTGAAAACCATGAAATTTCCGAGCAAA ACCGCCGAAGCGAAAGAAGAACGTAAAGTGGTTGGCACCGCGTAAGGAAAGGAAACCATGGAAACCCTGCTGAAACCGTTTCCGAGCCCGCTGCTG AGCATCCCGACCCCGAACATGTATAGCTTTAAACATAACAGCACCTTTCCGAATCCGACCAAACAGAAAGATAGCCGCAAATTTCATTATCGTAACAAA AGCAGCACCCATTTTTGCAGCTTTCTGGATCTGGCGCCGACCAGCAAACCGGAAAGCCTGGATGTGAATATCAGCTGGGTTGATACCGATCTGGATGG CGCCGAATTTGATGTGATTATCATTGGTACCGGTCCGGCCGGCCTGCGTCTGGCAGAACAGGTTAGCAAATATGGTATTAAAGTGTGCTGTGTTGATC CGAGCCCGCTGAGCATGTGGCCGAACAATTATGGCGTGTGGGTTGATGAATTTGAAAAACTGGGTCTGGAAGATTGCCTGGATCATAAATGGCCGGT GAGCTGTGTTCATATCAGCGATCATAAAACCAAATATCTGGATCGCCCGTATGGTCGCGTGAGCCGTAAAAAACTGAAACTGAAACTGCTGAACAGCT GCGTGGAAAACCGTGTTAAATTCTACAAAGCGAAAGTGCTGAAAGTTAAACATGAAGAATTTGAAAGCAGCATTGTGTGTGATGATGGCCGCAAAAT CAGCGGTAGCCTGATTGTTGATGCGAGCGGCTATGCCAGCGATTTTATCGAATATGATAAACCGCGTAATCATGGCTATCAGGTGGCGCATGGTATTC TGGCCGAAGTTGATAATCATCCGTTTGATCTGGATAAAATGATGCTGATGGATTGGCGCGATAGCCATCTGGGTAACGAACCGTATCTGCGTGTGAAA AATACCAAAGAACCGACCTTTCTGTATGCGATGCCGTTTGATCGCAATCTGGTGTTTCTGGAAGAAACCAGCCTGGTTAGCCGTCCGATGCTGAGCTAT ATGGAAGTGAAACGTCGCATGGTTGCCCGCCTGCGTCATCTGGGCATTAAAGTGCGCAGCGTTCTGGAAGAAGAAAAATGTGTGATCACGATGGGCG GCCCGCTGCCGCGTATTCCGCAGAACGTTATGGCGATCGGCGGTACCAGCGGCATTGTGCATCCGAGCAGCGGTTATATGGTTGCGCGCAGCATGGC GCTGGCACCGGTGCTGGCGGAAGCCATCGTTGAAAGCCTGGGCAGCACCCGCATGATTCGTGGTAGCCAGCTGTATCATCGCGTGTGGAATGGTCTG TGGCCGAGCGATCGTCGCCGTGTTCGTGAATGCTATTGTTTTGGCATGGAAACCTTATTAAAACTGGATCTGGAAGGTACCCGCCGTCTGTTTGATGC GTTTTTCGATGTTGATCCGAAATATTGGCATGGCTTTCTGAGCAGCCGCCTGAGCGTGAAAGAACTGGCCGTTCTGAGCCTGTACCTGTTTGGTCATGC GAGCAACCTGGCCCGTCTGGATATCGTGACCAAATGTACCGTGCCGCTGGTTAAACTGCTGGGCAATCTGGCGATTGAAAGCCTGTAAGGATCC

GAATTCAGGAGGATTACAAAATGTTTCATTATCGTAACAAAAGCAGCACCCATTTTTGCAGCTTTCTGGATCTGGCGCCGACCAGCAAACCGGAAAGC CTGGATGTGAATATCAGCTGGGTTGATACCGATCTGGATGGCGCCGAATTTGATGTGATTATCATTGGTACCGGTCCGGCCGGCCTGCGTCTGGCAGA ACAGGTTAGCAAATATGGTATTAAAGTGTGCTGTGTTGATCCGAGCCCGCTGAGCATGTGGCCGAACAATTATGGCGTGTGGGTTGATGAATTTGAA AAACTGGGTCTGGAAGATTGCCTGGATCATAAATGGCCGGTGAGCTGTGTTCATATCAGCGATCATAAAACCAAATATCTGGATCGCCCGTATGGTCG CGTGAGCCGTAAAAAACTGAAACTGAAACTGCTGAACAGCTGCGTGGAAAACCGTGTTAAATTCTACAAAGCGAAAGTGCTGAAAGTTAAACATGAA GAATTTGAAAGCAGCATTGTGTGTGATGATGGCCGCAAAATCAGCGGTAGCCTGATTGTTGATGCGAGCGGCTATGCCAGCGATTTTATCGAATATGA 
(Vector: pUCara ${ }^{3}$, Cloning site:

EcoRI/HindIII)

pUCara-CaCCS ${\mathrm{M} 40^{-}}^{-}$

CaZEP

(Vector: pUCara ${ }^{3}$,

Cloning site:

EcoRI/Xbal)
TAAACCGCGTAATCATGGCTATCAGGTGGCGCATGGTATTCTGGCCGAAGTTGATAATCATCCGTTTGATCTGGATAAAATGATGCTGATGGATTGGC GCGATAGCCATCTGGGTAACGAACCGTATCTGCGTGTGAAAAATACCAAAGAACCGACCTTTCTGTATGCGATGCCGTTTGATCGCAATCTGGTGTTTC TGGAAGAAACCAGCCTGGTTAGCCGTCCGATGCTGAGCTATATGGAAGTGAAACGTCGCATGGTTGCCCGCCTGCGTCATCTGGGCATTAAAGTGCG CAGCGTTCTGGAAGAAGAAAAATGTGTGATCACGATGGGCGGCCCGCTGCCGCGTATTCCGCAGAACGTTATGGCGATCGGCGGTACCAGCGGCAT GTGCATCCGAGCAGCGGTTATATGGTTGCGCGCAGCATGGCGCTGGCACCGGTGCTGGCGGAAGCCATCGTTGAAAGCCTGGGCAGCACCCGCATG ATTCGTGGTAGCCAGCTGTATCATCGCGTGTGGAATGGTCTGTGGCCGAGCGATCGTCGCCGTGTTCGTGAATGCTATTGTITTGGCATGGAAACCTT ATTAAAACTGGATCTGGAAGGTACCCGCCGTCTGTTTGATGCGTTTTTCGATGTTGATCCGAAATATTGGCATGGCTTTCTGAGCAGCCGCCTGAGCGT GAAAGAACTGGCCGTTCTGAGCCTGTACCTGTTTGGTCATGCGAGCAACCTGGCCCGTCTGGATATCGTGACCAAATGTACCGTGCCGCTGGTTAAAC TGCTGGGCAATCTGGCGATTGAAAGCCTGTAAGCTT

GAATTCAGGAGGATTACAAAATGTTTCATTATCGTAACAAAAGCAGCACCCATTTTTGCAGCTTTCTGGATCTGGCGCCGACCAGCAAACCGGAAAGC CTGGATGTGAATATCAGCTGGGTTGATACCGATCTGGATGGCGCCGAATTTGATGTGATTATCATTGGTACCGGTCCGGCCGGCCTGCGTCTGGCAGA ACAGGTTAGCAAATATGGTATTAAAGTGTGCTGTGTTGATCCGAGCCCGCTGAGCATGTGGCCGAACAATTATGGCGTGTGGGTTGATGAATTTGAA AAACTGGGTCTGGAAGATTGCCTGGATCATAAATGGCCGGTGAGCTGTGTTCATATCAGCGATCATAAAACCAAATATCTGGATCGCCCGTATGGTCG CGTGAGCCGTAAAAAACTGAAACTGAAACTGCTGAACAGCTGCGTGGAAAACCGTGTTAAATTCTACAAAGCGAAAGTGCTGAAAGTTAAACATGAA GAATTTGAAAGCAGCATTGTGTGTGATGATGGCCGCAAAATCAGCGGTAGCCTGATTGTTGATGCGAGCGGCTATGCCAGCGATTTTATCGAATATGA TAAACCGCGTAATCATGGCTATCAGGTGGCGCATGGTATTCTGGCCGAAGTTGATAATCATCCGTTTGATCTGGATAAAATGATGCTGATGGATTGGC GCGATAGCCATCTGGGTAACGAACCGTATCTGCGTGTGAAAAATACCAAAGAACCGACCTTTCTGTATGCGATGCCGTTTGATCGCAATCTGGTGTTTC TGGAAGAAACCAGCCTGGTTAGCCGTCCGATGCTGAGCTATATGGAAGTGAAACGTCGCATGGTTGCCCGCCTGCGTCATCTGGGCATTAAAGTGCG CAGCGTTCTGGAAGAAGAAAAATGTGTGATCACGATGGGCGGCCCGCTGCCGCGTATTCCGCAGAACGTTATGGCGATCGGCGGTACCAGCGGCATT GTGCATCCGAGCAGCGGTTATATGGTTGCGCGCAGCATGGCGCTGGCACCGGTGCTGGCGGAAGCCATCGTTGAAAGCCTGGGCAGCACCCGCATG ATTCGTGGTAGCCAGCTGTATCATCGCGTGTGGAATGGTCTGTGGCCGAGCGATCGTCGCCGTGTTCGTGAATGCTATTGTTTTGGCATGGAAACCTT ATTAAAACTGGATCTGGAAGGTACCCGCCGTCTGTTTGATGCGTTTTTCGATGTTGATCCGAAATATTGGCATGGCTTTCTGAGCAGCCGCCTGAGCGT GAAAGAACTGGCCGTTCTGAGCCTGTACCTGTTTGGTCATGCGAGCAACCTGGCCCGTCTGGATATCGTGACCAAATGTACCGTGCCGCTGGTTAAAC TGCTGGGCAATCTGGCGATTGAAAGCCTGTAAGCTTTTGATTTAATATAAGGAGGTAGAAAAATGACCCTGGCCGAAGCACCGGCCACCCCGACCGA AAAATCTAACAGCGAAGTGCCGCAGAAAAAACTGAAAGTGCTGGTTGCCGGCGGTGGCATTGGTGGCCTGGTGTTTGCGCTGGCGGCGAAAAAGAA AGGCTTTGATGTGCTGGTTTTTGAACGTGATCTGAGCGCCATCCGCGGCGAAGGCCAGTATCGTGGCCCGATTCAGATCCAGAGCAATGCCCTGGCG GCCCTGGAAGCGATTGATATGGATGTGGCCGAAGAAATCATGAACGCGGGTTGCATTACCGGCCAGCGTATCAATGGTCTGGTTGATGGTATTAGCG GCAACTGGTATTGTAAATTTGATACCTTTACCCCGGCCGTGGAACGCGGCCTGCCGGTGACCCGTGTTATTAGCCGCATGACCCTGCAGCAGATCCTG GCACGTGCCGTGGGTGAAGATGTTATTATGAACGAAAGCAATGTGGTTAACTTTGAAGATGATGGCGAAAAAGTGACCGTGGTTCTGGAAAATGGTC AGCGCTTTACCGGCGATCTGCTGGTTGGTGCCGATGGCATCCGTAGCAAAGTGCGCACCAACCTGTTTGGTCATAGCGAAGCGACCTATAGCGGTTAT ACCTGTTATACCGGCATTGCCGATTTTGTTCCGGCGGATATCGATACCGTGGGTTATCGTGTTTTTCTGGGCCATAAACAGTATTTTGTGAGCAGCGAT GTTGGTGGCGGTAAAATGCAGTGGTATGCGTTTCATAATGAACCGGCCGGCGGTGTGGATGCCCCGAATGGCAAGAAAGAACGCCTGCTGAAAATT TTGGCGGTTGGTGCGATAATGTGATCGATCTGCTGGTTGCCACCGATGAAGATGCGATTCTGCGTCGCGATATCTATGATCGTCCGCCGACCTTTAATT GGGGTCGTGGTCGTGTGACCCTGCTGGGTGATAGCGTTCATGCCATGCAGCCGAACCTGGGTCAGGGCGGTTGTATGGCCATTGAAGATAGCTATCA GCTGGCGCTGGAACTGGAAAAAGCCTGGAGCCGCAGCGCCGAAAGCGGTAGCCCGATGGATGTTATCAGCAGCCTGCGTAGCTATGAAAGCGCCCG TAAACTGCGCGTGGGTGTTATTCATGGCCTGGCCCGTATGGCCGCAATCATGGCAAGCACCTATAAAGCGTATCTGGGCGTGGGTCTGGGTCCGCTG AGCTTTCTGACCAAATATCGTATTCCGCACCCGGGTCGTGTTGGCGGTCGTGTGTTTGTTGATCTGGGCATGCCGCTGATGCTGAGCTGGGTGCTGGG CGGTAATGGTGATAAACTGGAAGGCCGTATCCAGCATTGCCGCCTGAGCGAAAAAGCCAACGATCAGCTGCGTCGCTGGTTTGAAGATGATGATGCG CTGGAACGCGCCACCGATGCCGAATGGCTGCTGCTGCCGGCCGCAAATGGCAATAGCGCGCTGGAAACCATTGTGCTGAGCCGTGATGAAGATGTTC CGTGCACCATTGGTAGCGTGAGCCATACCAACATCCCGGGCAAAAGCGTGGTTCTGCCGCTGCCGCAGGTTAGCGAAATGCATGCCCAGATTAGCTG TAAAAACAATGCGTTTTTCGTGACCGATTTTCAGAGCGAACATGGTACCTGGGTTATCGATAATGAAGGCCGTCGCTATCGCGTGAGCCCGAACTTTCC GATGCGTTTTCATAGCAGCGATGTGATTGTTTTTGGTAGCGATAAAGCGGCCTTTCGCGTGAAAACCATGAAATTTCCGAGCAAAACCGCCGAAGCGA AAGAAGAACGTAAAGTGGTTGGCACCGCGTAATCTAGA 
Table S5. DNA sequences of plasmids based on pUClac vector.

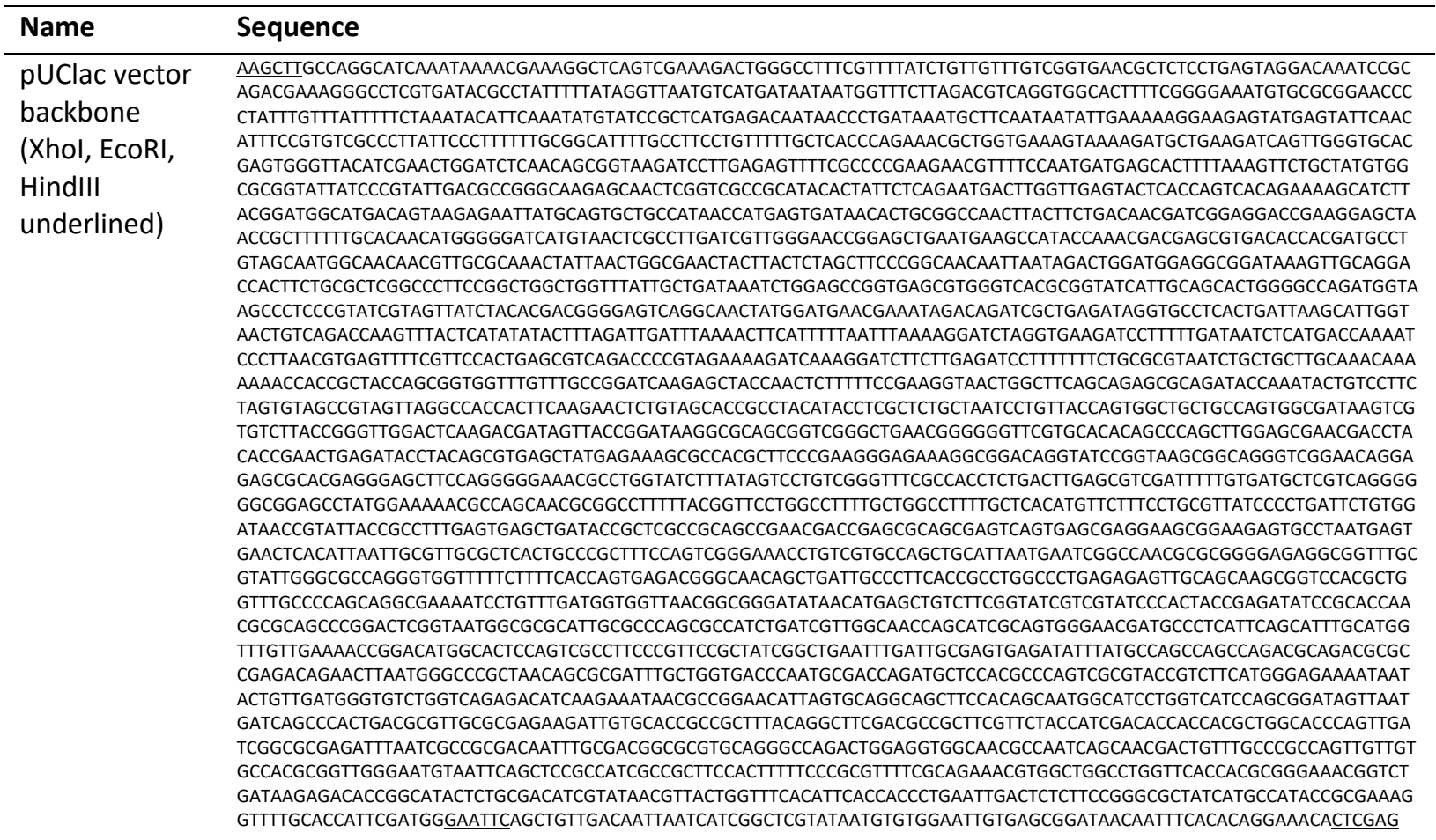

pUClac-CaZEP

(Vector:

pUClac,

Cloning site:

Xhol, HindIII)
pUClac-

CaZEP $_{\text {M56 }}$

(Vector:

pUClac,

Cloning site:

Xhol, HindIII)
pUClac-AtZEP

(Vector:

pUClac,

Cloning site:

Xhol, HindIII)
CTCGAGTCACACAGGAAACAGCTATGACCATGATTACGAATTCGATGTATAGCACCGTGTTTTATACCAGCGTTCATCCGAGCACCAGCGTGTTTAGCCGCAAACA GCTGCCGCTGCTGATTAGCAAAGATTTTCCGGCCGAACTGTATCATAGCCTGCCGTGCAAAAGCCTGGAAAATGGTCATATTAAGAAAGTGAAAGGCGTTAAAGC CACCCTGGCCGAAGCACCGGCCACCCCGACCGAAAAATCTAACAGCGAAGTGCCGCAGAAAAAACTGAAAGTGCTGGTTGCCGGCGGTGGCATTGGTGGCCTGG TGTTTGCGCTGGCGGCGAAAAAGAAAGGCTTTGATGTGCTGGTTTTTGAACGTGATCTGAGCGCCATCCGCGGCGAAGGCCAGTATCGTGGCCCGATTCAGATCC AGAGCAATGCCCTGGCGGCCCTGGAAGCGATTGATATGGATGTGGCCGAAGAAATCATGAACGCGGGTTGCATTACCGGCCAGCGTATCAATGGTCTGGTTGAT GGTATTAGCGGCAACTGGTATTGTAAATTTGATACCTTTACCCCGGCCGTGGAACGCGGCCTGCCGGTGACCCGTGTTATTAGCCGCATGACCCTGCAGCAGATCC TGGCACGTGCCGTGGGTGAAGATGTTATTATGAACGAAAGCAATGTGGTTAACTTTGAAGATGATGGCGAAAAAGTGACCGTGGTTCTGGAAAATGGTCAGCGC TTACCGGCGATCTGCTGGTTGGTGCCGATGGCATCCGTAGCAAAGTGCGCACCAACCTGTTTGGTCATAGCGAAGCGACCTATAGCGGTTATACCTGTTATACCGG CATTGCCGATTTTGTTCCGGCGGATATCGATACCGTGGGTTATCGTGTTTTTCTGGGCCATAAACAGTATTTTGTGAGCAGCGATGTTGGTGGCGGTAAAATGCAG TGGTATGCGTTTCATAATGAACCGGCCGGCGGTGTGGATGCCCCGAATGGCAAGAAAGAACGCCTGCTGAAAATTTTTGGCGGTTGGTGCGATAATGTGATCGAT CTGCTGGTTGCCACCGATGAAGATGCGATTCTGCGTCGCGATATCTATGATCGTCCGCCGACCTTTAATTGGGGTCGTGGTCGTGTGACCCTGCTGGGTGATAGCG TTCATGCCATGCAGCCGAACCTGGGTCAGGGCGGTTGTATGGCCATTGAAGATAGCTATCAGCTGGCGCTGGAACTGGAAAAAGCCTGGAGCCGCAGCGCCGAA AGCGGTAGCCCGATGGATGTTATCAGCAGCCTGCGTAGCTATGAAAGCGCCCGTAAACTGCGCGTGGGTGTTATTCATGGCCTGGCCCGTATGGCCGCAATCATG GCAAGCACCTATAAAGCGTATCTGGGCGTGGGTCTGGGTCCGCTGAGCTTTCTGACCAAATATCGTATTCCGCACCCGGGTCGTGTTGGCGGTCGTGTGTTTGTTG ATCTGGGCATGCCGCTGATGCTGAGCTGGGTGCTGGGCGGTAATGGTGATAAACTGGAAGGCCGTATCCAGCATTGCCGCCTGAGCGAAAAAGCCAACGATCAG CTGCGTCGCTGGTTTGAAGATGATGATGCGCTGGAACGCGCCACCGATGCCGAATGGCTGCTGCTGCCGGCCGCAAATGGCAATAGCGCGCTGGAAACCATTGTG CTGAGCCGTGATGAAGATGTTCCGTGCACCATTGGTAGCGTGAGCCATACCAACATCCCGGGCAAAAGCGTGGTTCTGCCGCTGCCGCAGGTTAGCGAAATGCAT GCCCAGATTAGCTGTAAAAACAATGCGTTTTCGTGACCGATTTTCAGAGCGAACATGGTACCTGGGTTATCGATAATGAAGGCCGTCGCTATCGCGTGAGCCCGA ACTTTCCGATGCGTTTTCATAGCAGCGATGTGATTGTTTTTGGTAGCGATAAAGCGGCCTTTCGCGTGAAAACCATGAAATTTCCGAGCAAAACCGCCGAAGCGAA AGAAGAACGTAAAGTGGTTGGCACCGCGTAAGCTT

CTCGAGTTGATTTAATATAAGGAGGTAGAAAAATGACCCTGGCCGAAGCACCGGCCACCCCGACCGAAAAATCTAACAGCGAAGTGCCGCAGAAAAAACTGAAA GTGCTGGTTGCCGGCGGTGGCATTGGTGGCCTGGTGTTTGCGCTGGCGGCGAAAAAGAAAGGCTTTGATGTGCTGGTTTTTGAACGTGATCTGAGCGCCATCCGC GGCGAAGGCCAGTATCGTGGCCCGATTCAGATCCAGAGCAATGCCCTGGCGGCCCTGGAAGCGATTGATATGGATGTGGCCGAAGAAATCATGAACGCGGGTTG CATTACCGGCCAGCGTATCAATGGTCTGGTTGATGGTATTAGCGGCAACTGGTATTGTAAATTTGATACCTTTACCCCGGCCGTGGAACGCGGCCTGCCGGTGACC CGTGTTATTAGCCGCATGACCCTGCAGCAGATCCTGGCACGTGCCGTGGGTGAAGATGTTATTATGAACGAAAGCAATGTGGTTAACTTTGAAGATGATGGCGAA AAAGTGACCGTGGTTCTGGAAAATGGTCAGCGCTTTACCGGCGATCTGCTGGTTGGTGCCGATGGCATCCGTAGCAAAGTGCGCACCAACCTGTTTGGTCATAGC GAAGCGACCTATAGCGGTTATACCTGTTATACCGGCATTGCCGATTTTGTTCCGGCGGATATCGATACCGTGGGTTATCGTGTTTTTCTGGGCCATAAACAGTATTT TGTGAGCAGCGATGTTGGTGGCGGTAAAATGCAGTGGTATGCGTTTCATAATGAACCGGCCGGCGGTGTGGATGCCCCGAATGGCAAGAAAGAACGCCTGCTGA AAATTTTGGCGGTTGGTGCGATAATGTGATCGATCTGCTGGTTGCCACCGATGAAGATGCGATTCTGCGTCGCGATATCTATGATCGTCCGCCGACCTTTAATTGG GGTCGTGGTCGTGTGACCCTGCTGGGTGATAGCGTTCATGCCATGCAGCCGAACCTGGGTCAGGGCGGTTGTATGGCCATTGAAGATAGCTATCAGCTGGCGCTG GAACTGGAAAAAGCCTGGAGCCGCAGCGCCGAAAGCGGTAGCCCGATGGATGTTATCAGCAGCCTGCGTAGCTATGAAAGCGCCCGTAAACTGCGCGTGGGTGT TATTCATGGCCTGGCCCGTATGGCCGCAATCATGGCAAGCACCTATAAAGCGTATCTGGGCGTGGGTCTGGGTCCGCTGAGCTTTCTGACCAAATATCGTATTCCG CACCCGGGTCGTGTTGGCGGTCGTGTGTTTGTTGATCTGGGCATGCCGCTGATGCTGAGCTGGGTGCTGGGCGGTAATGGTGATAAACTGGAAGGCCGTATCCAG CATTGCCGCCTGAGCGAAAAAGCCAACGATCAGCTGCGTCGCTGGTTTGAAGATGATGATGCGCTGGAACGCGCCACCGATGCCGAATGGCTGCTGCTGCCGGCC GCAAATGGCAATAGCGCGCTGGAAACCATTGTGCTGAGCCGTGATGAAGATGTTCCGTGCACCATTGGTAGCGTGAGCCATACCAACATCCCGGGCAAAAGCGTG GTTCTGCCGCTGCCGCAGGTTAGCGAAATGCATGCCCAGATTAGCTGTAAAAACAATGCGTTTTTCGTGACCGATTTTCAGAGCGAACATGGTACCTGGGTTATCG ATAATGAAGGCCGTCGCTATCGCGTGAGCCCGAACTTTCCGATGCGTTTTCATAGCAGCGATGTGATTGTTTTTGGTAGCGATAAAGCGGCCTTTCGCGTGAAAAC CATGAAATTTCCGAGCAAAACCGCCGAAGCGAAAGAAGAACGTAAAGTGGTTGGCACCGCGTAAAAGCTT

CTCGAGTCACACAGGAAACAGCTATGACCATGATTACGAATTCGATGGGTAGCACCCCGTTTTGCTATAGCATCAACCCGAGCCCGAGCAAACTGGATTTTACCCG CACCCATGTGTTTAGCCCGGTTAGCAAACAGTTTTATCTGGATCTGAGCAGCTTTAGCGGTAAACCGGGCGGTGTGAGCGGTTTTCGTAGCCGTCGCGCCCTGCTG GGCGTTAAAGCCGCAACCGCCCTGGTGGAAAAAGAAGAAAAACGTGAAGCGGTTACCGAAAAGAAAAAGAAAAGCCGTGTGCTGGTTGCGGGCGGTGGCATCG GTGGCCTGGTGTTTGCCCTGGCGGCGAAAAAGAAAGGCTTTGATGTGCTGGTTTTTGAAAAAGATCTGAGCGCGATTCGTGGCGAAGGCAAATATCGCGGCCCG ATTCAGATTCAGAGCAATGCCCTGGCCGCACTGGAAGCCATTGATATCGAAGTGGCGGAACAGGTTATGGAAGCCGGTTGCATCACCGGCGATCGCATTAATGGT CTGGTTGATGGTATTAGCGGCACCTGGTATGTGAAATTTGATACCTTTACCCCGGCCGCAAGCCGTGGCCTGCCGGTGACCCGTGTTATCAGCCGCATGACCCTGC AGCAGATTCTGGCGCGTGCCGTGGGTGAAGATGTTATCCGCAACGAAAGCAATGTGGTTGATTTTGAAGATAGCGGCGATAAAGTGACCGTGGTTCTGGAAAAT GGTCAGCGCTATGAAGGCGATCTGCTGGTTGGTGCGGATGGCATTTGGAGCAAAGTGCGTAACAACCTGTTTGGTCGCAGCGAAGCCACCTATAGCGGTTATACC TGTTATACCGGCATTGCGGATTTTATCCCGGCCGATATTGAAAGCGTGGGTTATCGCGTTTTTTCTGGGCCATAAACAGTATTTTGTGAGCAGCGATGTTGGTGGCG 
GTAAAATGCAGTGGTATGCGTTTCATGAAGAACCGGCCGGCGGTGCGGATGCACCGAACGGTATGAAAAAACGTCTGTTCGAAATCTTCGATGGCTGGTGCGATA ATGTTCTGGATCTGCTGCATGCGACCGAAGAAGAAGCGATCCTGCGTCGCGATATTTATGATCGTAGCCCGGGCTTTACCTGGGGCAAAGGTCGCGTGACCCTGCT GGGTGATAGCATCCATGCGATGCAGCCGAACATGGGCCAGGGCGGTTGTATGGCCATTGAAGATAGCTTTCAGCTGGCGCTGGAACTGGATGAAGCCTGGAAAC AGAGCGTGGAAACCACCACCCCGGTTGATGTGGTTAGCAGCCTGAAACGTTATGAAGAAAGCCGTCGCCTGCGCGTTGCGATTATCCATGCGATGGCCCGTATGG CCGCAATCATGGCCAGCACCTATAAAGCCTATCTGGGCGTGGGTCTGGGTCCGCTGAGCTTTCTGACCAAATTTCGTGTGCCGCATCCGGGTCGTGTGGGCGGCCG TTTCTTTGTGGATATTGCGATGCCGAGCATGCTGGATTGGGTGCTGGGCGGTAATAGCGAAAAACTGCAGGGCCGTCCGCCGAGCTGTCGTCTGACCGATAAAGC GGATGATCGTCTGCGCGAATGGTTTGAAGATGATGATGCCCTGGAACGTACCATCAAGGGTGAATGGTATCTGATTCCGCATGGCGATGATTGCTGTGTTAGCGA AACCCTGTGCCTGACCAAAGATGAAGATCAGCCGTGTATCGTGGGTAGCGAACCGGATCAGGATTTTCCGGGCATGCGTATTGTTATCCCGAGCAGCCAGGTGAG CAAAATGCATGCGCGCGTTATTTATAAAGATGGTGCGTTTTTCCTGATGGATCTGCGTAGCGAACATGGTACCTATGTGACCGATAACGAAGGCCGTCGCTATCGC GCGACCCCGAATTTTCCGGCCCGTTTTCGCAGCAGCGATATTATCGAATTTGGCAGCGATAAGAAAGCGGCCTTTCGTGTGAAAGTTATTCGCAAAACCCCGAAAA GCACCCGTAAAAATGAAAGCAACAATGATAAACTGCTGCAGACCGCGTAAGCTT

pUClacAtZEP (Vector: pUClac, Cloning site: Xhol, HindIII)

CaCCS (Inserted into pUC-[ZEP]. Cloning site: Xhol; note that the one in $5^{\prime}$ end is partial sequence)

$\mathrm{CaCCS}_{\mathrm{M} 40}$ (Inserted into pUC-[ZEP]. Cloning site: Xhol; note that the one in $5^{\prime}$ end is partial sequence)

$\mathrm{P}_{\mathrm{T7}}-\mathrm{CaCCS}_{\mathrm{M} 40}$ (Inserted into pUC-CaZEP. Cloning site: EcoRl, Xhol: T7/lac promoter indicated by red color)

CTCGAGATATAAGGAGGTAGAAAAATGGCCGCAACCGCCCTGGTGGAAAAAGAAGAAAAACGTGAAGCGGTTACCGAAAAGAAAAAGAAAAGCCGTGTGCTGG TTGCGGGCGGTGGCATCGGTGGCCTGGTGTTTGCCCTGGCGGCGAAAAAGAAAGGCTTTGATGTGCTGGTTTTTGAAAAAGATCTGAGCGCGATTCGTGGCGAA GGCAAATATCGCGGCCCGATTCAGATTCAGAGCAATGCCCTGGCCGCACTGGAAGCCATTGATATCGAAGTGGCGGAACAGGTTATGGAAGCCGGTTGCATCACC GGCGATCGCATTAATGGTCTGGTTGATGGTATTAGCGGCACCTGGTATGTGAAATTTGATACCTTTACCCCGGCCGCAAGCCGTGGCCTGCCGGTGACCCGTGTTA TCAGCCGCATGACCCTGCAGCAGATTCTGGCGCGTGCCGTGGGTGAAGATGTTATCCGCAACGAAAGCAATGTGGTTGATTTTGAAGATAGCGGCGATAAAGTGA CCGTGGTTCTGGAAAATGGTCAGCGCTATGAAGGCGATCTGCTGGTTGGTGCGGATGGCATTTGGAGCAAAGTGCGTAACAACCTGTTTGGTCGCAGCGAAGCCA CCTATAGCGGTTATACCTGTTATACCGGCATTGCGGATTTTATCCCGGCCGATATTGAAAGCGTGGGTTATCGCGTTTTTCTGGGCCATAAACAGTATTTTGTGAGC AGCGATGTTGGTGGCGGTAAAATGCAGTGGTATGCGTTTCATGAAGAACCGGCCGGCGGTGCGGATGCACCGAACGGTATGAAAAAACGTCTGTTCGAAATCTT CGATGGCTGGTGCGATAATGTTCTGGATCTGCTGCATGCGACCGAAGAAGAAGCGATCCTGCGTCGCGATATTTATGATCGTAGCCCGGGCTTTACCTGGGGCAA AGGTCGCGTGACCCTGCTGGGTGATAGCATCCATGCGATGCAGCCGAACATGGGCCAGGGCGGTTGTATGGCCATTGAAGATAGCTTTCAGCTGGCGCTGGAACT GGATGAAGCCTGGAAACAGAGCGTGGAAACCACCACCCCGGTTGATGTGGTTAGCAGCCTGAAACGTTATGAAGAAAGCCGTCGCCTGCGCGTTGCGATTATCCA TGCGATGGCCCGTATGGCCGCAATCATGGCCAGCACCTATAAAGCCTATCTGGGCGTGGGTCTGGGTCCGCTGAGCTTTCTGACCAAATTTCGTGTGCCGCATCCG GGTCGTGTGGGCGGCCGTTTCTTTGTGGATATTGCGATGCCGAGCATGCTGGATTGGGTGCTGGGCGGTAATAGCGAAAAACTGCAGGGCCGTCCGCCGAGCTG TCGTCTGACCGATAAAGCGGATGATCGTCTGCGCGAATGGTTTGAAGATGATGATGCCCTGGAACGTACCATCAAGGGTGAATGGTATCTGATTCCGCATGGCGA TGATTGCTGTGTTAGCGAAACCCTGTGCCTGACCAAAGATGAAGATCAGCCGTGTATCGTGGGTAGCGAACCGGATCAGGATTTTCCGGGCATGCGTATTGTTATC CCGAGCAGCCAGGTGAGCAAAATGCATGCGCGCGTTATTTATAAAGATGGTGCGTTTTTCCTGATGGATCTGCGTAGCGAACATGGTACCTATGTGACCGATAAC GAAGGCCGTCGCTATCGCGCGACCCCGAATTTTCCGGCCCGTTTTCGCAGCAGCGATATTATCGAATTTGGCAGCGATAAGAAAGCGGCCTTTCGTGTGAAAGTTA TTCGCAAAACCCCGAAAAGCACCCGTAAAAATGAAAGCAACAATGATAAACTGCTGCAGACCGCGTAAGCTT

CTCGATCTAGAGGAGGTATATTTATGGAAACCCTGCTGAAACCGTTTCCGAGCCCGCTGCTGAGCATCCCGACCCCGAACATGTATAGCTTTAAACATAACAGCAC CTTTCCGAATCCGACCAAACAGAAAGATAGCCGCAAATTTCATTATCGTAACAAAAGCAGCACCCATTTTTGCAGCTTTCTGGATCTGGCGCCGACCAGCAAACCG GAAAGCCTGGATGTGAATATCAGCTGGGTTGATACCGATCTGGATGGCGCCGAATTTGATGTGATTATCATTGGTACCGGTCCGGCCGGCCTGCGTCTGGCAGAA CAGGTTAGCAAATATGGTATTAAAGTGTGCTGTGTTGATCCGAGCCCGCTGAGCATGTGGCCGAACAATTATGGCGTGTGGGTTGATGAATTTGAAAAACTGGGT CTGGAAGATTGCCTGGATCATAAATGGCCGGTGAGCTGTGTTCATATCAGCGATCATAAAACCAAATATCTGGATCGCCCGTATGGTCGCGTGAGCCGTAAAAAAC TGAAACTGAAACTGCTGAACAGCTGCGTGGAAAACCGTGTTAAATTCTACAAAGCGAAAGTGCTGAAAGTTAAACATGAAGAATTTGAAAGCAGCATTGTGTGTG ATGATGGCCGCAAAATCAGCGGTAGCCTGATTGTTGATGCGAGCGGCTATGCCAGCGATTTTATCGAATATGATAAACCGCGTAATCATGGCTATCAGGTGGCGC ATGGTATTCTGGCCGAAGTTGATAATCATCCGTTTGATCTGGATAAAATGATGCTGATGGATTGGCGCGATAGCCATCTGGGTAACGAACCGTATCTGCGTGTGAA AAATACCAAAGAACCGACCTTTCTGTATGCGATGCCGTTTGATCGCAATCTGGTGTTTCTGGAAGAAACCAGCCTGGTTAGCCGTCCGATGCTGAGCTATATGGAA GTGAAACGTCGCATGGTTGCCCGCCTGCGTCATCTGGGCATTAAAGTGCGCAGCGTTCTGGAAGAAGAAAAATGTGTGATCACGATGGGCGGCCCGCTGCCGCGT ATTCCGCAGAACGTTATGGCGATCGGCGGTACCAGCGGCATTGTGCATCCGAGCAGCGGTTATATGGTTGCGCGCAGCATGGCGCTGGCACCGGTGCTGGCGGA AGCCATCGTTGAAAGCCTGGGCAGCACCCGCATGATTCGTGGTAGCCAGCTGTATCATCGCGTGTGGAATGGTCTGTGGCCGAGCGATCGTCGCCGTGTTCGTGA ATGCTATTGTTTTGGCATGGAAACCTTATTAAAACTGGATCTGGAAGGTACCCGCCGTCTGTTTGATGCGTTTTTCGATGTTGATCCGAAATATTGGCATGGCTTTC AAGCAGCCGCCTGAGCGTGAAAGAACTGGCCGTTCTGAGCCTGTACCTGTTTGGTCATGCGAGCAACCTGGCCCGTCTGGATATCGTGACCAAATGTACCGTGCC GCTGGTTAAACTGCTGGGCAATCTGGCGATTGAAAGCCTGTAACTCGAG

CTCGACTAGAGGAGGATTACAAAATGTTTCATTATCGTAACAAAAGCAGCACCCATTTTTGCAGCTTTCTGGATCTGGCGCCGACCAGCAAACCGGAAAGCCTGGA TGTGAATATCAGCTGGGTTGATACCGATCTGGATGGCGCCGAATTTGATGTGATTATCATTGGTACCGGTCCGGCCGGCCTGCGTCTGGCAGAACAGGTTAGCAA ATATGGTATTAAAGTGTGCTGTGTTGATCCGAGCCCGCTGAGCATGTGGCCGAACAATTATGGCGTGTGGGTTGATGAATTTGAAAAACTGGGTCTGGAAGATTG CCTGGATCATAAATGGCCGGTGAGCTGTGTTCATATCAGCGATCATAAAACCAAATATCTGGATCGCCCGTATGGTCGCGTGAGCCGTAAAAAACTGAAACTGAAA CTGCTGAACAGCTGCGTGGAAAACCGTGTTAAATTCTACAAAGCGAAAGTGCTGAAAGTTAAACATGAAGAATTTGAAAGCAGCATTGTGTGTGATGATGGCCGC AAAATCAGCGGTAGCCTGATTGTTGATGCGAGCGGCTATGCCAGCGATTTTATCGAATATGATAAACCGCGTAATCATGGCTATCAGGTGGCGCATGGTATTCTGG CCGAAGTTGATAATCATCCGTTTGATCTGGATAAAATGATGCTGATGGATTGGCGCGATAGCCATCTGGGTAACGAACCGTATCTGCGTGTGAAAAATACCAAAGA ACCGACCTTTCTGTATGCGATGCCGTTTGATCGCAATCTGGTGTTTCTGGAAGAAACCAGCCTGGTTAGCCGTCCGATGCTGAGCTATATGGAAGTGAAACGTCGC ATGGTTGCCCGCCTGCGTCATCTGGGCATTAAAGTGCGCAGCGTTCTGGAAGAAGAAAAATGTGTGATCACGATGGGCGGCCCGCTGCCGCGTATTCCGCAGAAC GTTATGGCGATCGGCGGTACCAGCGGCATTGTGCATCCGAGCAGCGGTTATATGGTTGCGCGCAGCATGGCGCTGGCACCGGTGCTGGCGGAAGCCATCGTTGA AAGCCTGGGCAGCACCCGCATGATTCGTGGTAGCCAGCTGTATCATCGCGTGTGGAATGGTCTGTGGCCGAGCGATCGTCGCCGTGTTCGTGAATGCTATTGTTTI GGCATGGAAACCTTATTAAAACTGGATCTGGAAGGTACCCGCCGTCTGTTTGATGCGTTTTTCGATGTTGATCCGAAATATTGGCATGGCTTTCTGAGCAGCCGCC GAGCGTGAAAGAACTGGCCGTTCTGAGCCTGTACCTGTTTGGTCATGCGAGCAACCTGGCCCGTCTGGATATCGTGACCAAATGTACCGTGCCGCTGGTTAAACTG CTGGGCAATCTGGCGATTGAAAGCCTGTAACTCGAG

GAATTCTAATACGACTCACTATAGGGGAATTGTGAGCGGATAACAATTCCCCTCTAGAGGAGGATTACAAAATGCATCATCACCATCACCACTTTCATTATCGTAAC AAAAGCAGCACCCATTTTTGCAGCTTTCTGGATCTGGCGCCGACCAGCAAACCGGAAAGCCTGGATGTGAATATCAGCTGGGTTGATACCGATCTGGATGGCGCC GAATTTGATGTGATTATCATTGGTACCGGTCCGGCCGGCCTGCGTCTGGCAGAACAGGTTAGCAAATATGGTATTAAAGTGTGCTGTGTTGATCCGAGCCCGCTGA GCATGTGGCCGAACAATTATGGCGTGTGGGTTGATGAATTTGAAAAACTGGGTCTGGAAGATTGCCTGGATCATAAATGGCCGGTGAGCTGTGTTCATATCAGCG ATCATAAAACCAAATATCTGGATCGCCCGTATGGTCGCGTGAGCCGTAAAAAACTGAAACTGAAACTGCTGAACAGCTGCGTGGAAAACCGTGTTAAATTCTACAA AGCGAAAGTGCTGAAAGTTAAACATGAAGAATTTGAAAGCAGCATTGTGTGTGATGATGGCCGCAAAATCAGCGGTAGCCTGATTGTTGATGCGAGCGGCTATGC CAGCGATTTTATCGAATATGATAAACCGCGTAATCATGGCTATCAGGTGGCGCATGGTATTCTGGCCGAAGTTGATAATCATCCGTTTGATCTGGATAAAATGATG CTGATGGATTGGCGCGATAGCCATCTGGGTAACGAACCGTATCTGCGTGTGAAAAATACCAAAGAACCGACCTTTCTGTATGCGATGCCGTTTGATCGCAATCTGG TGTTTCTGGAAGAAACCAGCCTGGTTAGCCGTCCGATGCTGAGCTATATGGAAGTGAAACGTCGCATGGTTGCCCGCCTGCGTCATCTGGGCATTAAAGTGCGCA GCGTTCTGGAAGAAGAAAAATGTGTGATCACGATGGGCGGCCCGCTGCCGCGTATTCCGCAGAACGTTATGGCGATCGGCGGTACCAGCGGCATTGTGCATCCG AGCAGCGGTTATATGGTTGCGCGCAGCATGGCGCTGGCACCGGTGCTGGCGGAAGCCATCGTTGAAAGCCTGGGCAGCACCCGCATGATTCGTGGTAGCCAGCT GTATCATCGCGTGTGGAATGGTCTGTGGCCGAGCGATCGTCGCCGTGTTCGTGAATGCTATTGTTTTGGCATGGAAACCTTATTAAAACTGGATCTGGAAGGTACC CGCCGTCTGTTTGATGCGTTTTTCGATGTTGATCCGAAATATTGGCATGGCTTTCTGAGCAGCCGCCTGAGCGTGAAAGAACTGGCCGTTCTGAGCCTGTACCTGTT TGGTCATGCGAGCAACCTGGCCCGTCTGGATATCGTGACCAAATGTACCGTGCCGCTGGTTAAACTGCTGGGCAATCTGGCGATTGAAAGCCTGTAACTCGAG 


\section{Supporting References}

1. Takemura, M., Maoka, T. \& Misawa, N. Biosynthetic routes of hydroxylated carotenoids (xanthophylls) in Marchantia polymorpha, and production of novel and rare xanthophylls through pathway engineering in Escherichia coli. Planta 241, 699-710 (2015).

2. Misawa, N. et al. Structure and functional analysis of a marine bacterial carotenoid biosynthesis gene cluster and astaxanthin biosynthetic pathway proposed at the gene level. Journal of Bacteriology 177, 6575-6584 (1995).

3. Furubayashi, M. et al. A High-Throughput Colorimetric Screening Assay for Terpene Synthase Activity Based on Substrate Consumption. PLoS ONE 9, e93317 (2014). 